\title{
Global Dynamics of a Competitive System of Rational Difference Equations in the Plane
}

\author{
S. Kalabušić, ${ }^{1}$ M. R. S. Kulenović, ${ }^{2}$ and E. Pilav ${ }^{1}$ \\ ${ }^{1}$ Department of Mathematics, University of Sarajevo, 71000 Sarajevo, Bosnia and Herzegovina \\ ${ }^{2}$ Department of Mathematics, University of Rhode Island, Kingston, RI 02881-0816, USA
}

Correspondence should be addressed to M. R. S. Kulenović, kulenm@math.uri.edu

Received 26 August 2009; Accepted 8 December 2009

Recommended by Panayiotis Siafarikas

We investigate global dynamics of the following systems of difference equations $x_{n+1}=\left(\alpha_{1}+\right.$ $\left.\beta_{1} x_{n}\right) / y_{n}, y_{n+1}=\left(\alpha_{2}+\gamma_{2} y_{n}\right) /\left(A_{2}+x_{n}\right), n=0,1,2, \ldots$, where the parameters $\alpha_{1}, \beta_{1}, \alpha_{2}, \gamma_{2}$, and $A_{2}$ are positive numbers and initial conditions $x_{0}$ and $y_{0}$ are arbitrary nonnegative numbers such that $y_{0}>0$. We show that this system has rich dynamics which depend on the part of parametric space. We show that the basins of attractions of different locally asymptotically stable equilibrium points are separated by the global stable manifolds of either saddle points or of nonhyperbolic equilibrium points.

Copyright (C) 2009 S. Kalabušić et al. This is an open access article distributed under the Creative Commons Attribution License, which permits unrestricted use, distribution, and reproduction in any medium, provided the original work is properly cited.

\section{Introduction and Preliminaries}

In this paper, we study the global dynamics of the following rational system of difference equations:

$$
\begin{aligned}
& x_{n+1}=\frac{\alpha_{1}+\beta_{1} x_{n}}{y_{n}}, \\
& y_{n+1}=\frac{\alpha_{2}+\gamma_{2} y_{n}}{A_{2}+x_{n}}, \quad n=0,1,2, \ldots,
\end{aligned}
$$

where the parameters $\alpha_{1}, \beta_{1}, \alpha_{2}, \gamma_{2}$, and $A_{2}$ are positive numbers and initial conditions $x_{0} \geq$ 0 and $y_{0}>0$ are arbitrary numbers. System (1.1) was mentioned in [1] as a part of Open Problem 3 which asked for a description of global dynamics of three specific competitive systems. According to the labeling in [1], system (1.1) is called $(21,29)$. In this paper, we provide the precise description of global dynamics of system (1.1). We show that system 
(1.1) has a variety of dynamics that depend on the value of parameters. We show that system (1.1) may have between zero and two equilibrium points, which may have different local character. If system (1.1) has one equilibrium point, then this point is either locally saddle point or non-hyperbolic. If system (1.1) has two equilibrium points, then the pair of points is the pair of a saddle point and a sink. The major problem is determining the basins of attraction of different equilibrium points. System (1.1) gives an example of semistable non-hyperbolic equilibrium point. The typical results are Theorems 4.1 and 4.5 below.

System (1.1) is a competitive system, and our results are based on recent results developed for competitive systems in the plane; see $[2,3]$. In the next section, we present some general results about competitive systems in the plane. The third section deals with some basic facts such as the non-existence of period-two solution of system (1.1). The fourth section analyzes local stability which is fairly complicated for this system. Finally, the fifth section gives global dynamics for all values of parameters.

Let $\supset$ and $\partial$ be intervals of real numbers. Consider a first-order system of difference equations of the form

$$
\begin{aligned}
& x_{n+1}=f\left(x_{n}, y_{n}\right), \quad n=0,1,2, \ldots, \\
& y_{n+1}=g\left(x_{n}, y_{n}\right),
\end{aligned}
$$

where $f: 0 \times 2 \rightarrow 0, g: 0 \times 2 \rightarrow 2$, and $\left(x_{0}, y_{0}\right) \in 0 \times 2$.

When the function $f(x, y)$ is increasing in $x$ and decreasing in $y$ and the function $g(x, y)$ is decreasing in $x$ and increasing in $y$, the system (1.2) is called competitive. When the function $f(x, y)$ is increasing in $x$ and increasing in $y$ and the function $g(x, y)$ is increasing in $x$ and increasing in $y$, the system (1.2) is called cooperative. A map $T$ that corresponds to the system (1.2) is defined as $T(x, y)=(f(x, y), g(x, y))$. Competitive and cooperative maps, which are called monotone maps, are defined similarly. Strongly competitive systems of difference equations or maps are those for which the functions $f$ and $g$ are coordinate-wise strictly monotone.

If $\mathbf{v}=(u, v) \in \mathbb{R}^{2}$, we denote with $\mathcal{Q}_{\ell}(\mathbf{v}), \ell \in\{1,2,3,4\}$, the four quadrants in $\mathbb{R}^{2}$ relative to $\mathbf{v}$, that is, $Q_{1}(\mathbf{v})=\left\{(x, y) \in \mathbb{R}^{2}: x \geq u, y \geq v\right\}, Q_{2}(\mathbf{v})=\left\{(x, y) \in \mathbb{R}^{2}: x \leq u, y \geq\right.$ $v\}$, and so on. Define the South-East partial order $\preceq_{\text {se }}$ on $\mathbb{R}^{2}$ by $(x, y) \varliminf_{\mathrm{se}}(s, t)$ if and only if $x \leq s$ and $y \geq t$. Similarly, we define the North-East partial order $\leq_{\text {ne }}$ on $\mathbb{R}^{2}$ by $(x, y) \leq_{\text {ne }}(s, t)$ if and only if $x \leq s$ and $y \leq t$. For $\mathcal{A} \subset \mathbb{R}^{2}$ and $x \in \mathbb{R}^{2}$, define the distance from $x$ to $\mathcal{A}$ as $\operatorname{dist}(x, \mathcal{A}):=\inf \{\|x-y\|: y \in \mathcal{A}\}$. By int $\mathscr{A}$, we denote the interior of a set $\mathcal{A}$.

It is easy to show that a map $F$ is competitive if it is nondecreasing with respect to the South-East partial order, that is if the following holds:

$$
\left(\begin{array}{l}
x^{1} \\
y^{1}
\end{array}\right) \preceq_{\mathrm{se}}\left(\begin{array}{l}
x^{2} \\
y^{2}
\end{array}\right) \Longrightarrow F\left(\begin{array}{l}
x^{1} \\
y^{1}
\end{array}\right) \preceq_{\mathrm{se}} F\left(\begin{array}{l}
x^{2} \\
y^{2}
\end{array}\right)
$$

Competitive systems were studied by many authors; see [4-19], and others. All known results, with the exception of $[4,6,10]$, deal with hyperbolic dynamics. The results presented here are results that hold in both the hyperbolic and the non-hyperbolic cases.

We now state three results for competitive maps in the plane. The following definition is from [18]. 
Definition 1.1. Let $R$ be a nonempty subset of $\mathbb{R}^{2}$. A competitive map $T: R \rightarrow R$ is said to satisfy condition $(O+)$ if for every $x, y$ in $R, T(x) \preceq_{\mathrm{ne}} T(y)$ implies $x \preceq_{\mathrm{ne}} y$, and $T$ is said to satisfy condition $(O-)$ if for every $x, y$ in $R, T(x) \preceq_{\mathrm{ne}} T(y)$ implies $y \preceq_{\mathrm{ne}} x$.

The following theorem was proved by de Mottoni and Schiaffino [20] for the Poincaré map of a periodic competitive Lotka-Volterra system of differential equations. Smith generalized the proof to competitive and cooperative maps $[15,16]$.

Theorem 1.2. Let $R$ be a nonempty subset of $\mathbb{R}^{2}$. If $T$ is a competitive map for which $(O+)$ holds, then for all $x \in R,\left\{T^{n}(x)\right\}$ is eventually componentwise monotone. If the orbit of $x$ has compact closure, then it converges to a fixed point of $T$. If instead (O-) holds, then for all $x \in R,\left\{T^{2 n}\right\}$ is eventually componentwise monotone. If the orbit of $x$ has compact closure in $R$, then its omega limit set is either a period-two orbit or a fixed point.

The following result is from [18], with the domain of the map specialized to be the Cartesian product of intervals of real numbers. It gives a sufficient condition for conditions $(\mathrm{O}+)$ and $(\mathrm{O}-)$.

Theorem 1.3 (Smith [18]). Let $R \subset \mathbb{R}^{2}$ be the Cartesian product of two intervals in $\mathbb{R}$. Let $T: R \rightarrow$ $R$ be a $C^{1}$ competitive map. If $T$ is injective and $\operatorname{det} J_{T}(x)>0$ for all $x \in R$, then $T$ satisfies $(O+)$. If $T$ is injective and $\operatorname{det} J_{T}(x)<0$ for all $x \in R$, then $T$ satisfies $(O-)$.

Theorem 1.4. Let $T$ be a monotone map on a closed and bounded rectangular region $\mathbb{R} \subset \mathbb{R}^{2}$. Suppose that $T$ has a unique fixed point $\overline{\mathbf{e}}$ in $\boldsymbol{R}$. Then $\overline{\mathbf{e}}$ is a global attractor of $T$ on $\boldsymbol{R}$.

The following theorems were proved by Kulenović and Merino [3] for competitive systems in the plane, when one of the eigenvalues of the linearized system at an equilibrium (hyperbolic or non-hyperbolic) is by absolute value smaller than 1 while the other has an arbitrary value. These results are useful for determining basins of attraction of fixed points of competitive maps.

Our first result gives conditions for the existence of a global invariant curve through a fixed point (hyperbolic or not) of a competitive map that is differentiable in a neighborhood of the fixed point, when at least one of two nonzero eigenvalues of the Jacobian matrix of the map at the fixed point has absolute value less than one. A region $R \subset \mathbb{R}^{2}$ is rectangular if it is the Cartesian product of two intervals in $\mathbb{R}$.

Theorem 1.5. Let $T$ be a competitive map on a rectangular region $R \subset \mathbb{R}^{2}$. Let $\bar{x} \in \mathcal{R}$ be a fixed point of $T$ such that $\Delta:=\mathcal{R} \cap \operatorname{int}\left(Q_{1}(\bar{x}) \cup Q_{3}(\bar{x})\right)$ is nonempty (i.e., $\bar{x}$ is not the NW or SE vertex of $\mathcal{R}$ ), and $T$ is strongly competitive on $\Delta$. Suppose that the following statements are true.

(a) The map $T$ has a $C^{1}$ extension to a neighborhood of $\bar{x}$.

(b) The Jacobian matrix of $T$ at $\bar{x}$ has real eigenvalues $\lambda$, $\mu$ such that $0<|\lambda|<\mu$, where $|\lambda|<1$, and the eigenspace $E^{\mathfrak{\lambda}}$ associated with $\lambda$ is not a coordinate axis.

Then there exists a curve $\mathcal{C} \subset \mathcal{R}$ through $\bar{x}$ that is invariant and a subset of the basin of attraction of $\bar{x}$, such that $\mathcal{C}$ is tangential to the eigenspace $E^{\lambda}$ at $\bar{x}$, and $\mathcal{C}$ is the graph of a strictly increasing continuous function of the first coordinate on an interval. Any endpoints of $\mathcal{C}$ in the interior of $\mathcal{R}$ are either fixed points or minimal period-two points. In the latter case, the set of endpoints of $\mathcal{C}$ is a minimal period-two orbit of $T$. 
Corollary 1.6. If $T$ has no fixed point nor periodic points of minimal period-two in $\Delta$, then the endpoints of $\mathcal{C}$ belong to $\partial R$.

For maps that are strongly competitive near the fixed point, hypothesis $b$. of Theorem 1.5 reduces just to $|\lambda|<1$. This follows from a change of variables [18] that allows the Perron-Frobenius Theorem to be applied to give that, at any point, the Jacobian matrix of a strongly competitive map has two real and distinct eigenvalues, the larger one in absolute value being positive, and that corresponding eigenvectors may be chosen to point in the direction of the second and first quadrants, respectively. Also, one can show that in such case no associated eigenvector is aligned with a coordinate axis.

The following result gives a description of the global stable and unstable manifolds of a saddle point of a competitive map. The result is the modification of Theorem 1.7 from [12].

Theorem 1.7. In addition to the hypotheses of Theorem 1.5, suppose that $\mu>1$ and that the eigenspace $E^{\mu}$ associated with $\mu$ is not a coordinate axis. If the curve $\mathcal{C}$ of Theorem 1.5 has endpoints in $\partial \mathcal{R}$, then $\mathcal{C}$ is the global stable manifold $\mathcal{W}^{s}(\bar{x})$ of $\bar{x}$, and the global unstable manifold $\mathcal{W}^{u}(\bar{x})$ is a curve in $R$ that is tangential to $E^{\mu}$ at $\bar{x}$ and such that it is the graph of a strictly decreasing function of the first coordinate on an interval. Any endpoints of $W^{u}(\bar{x})$ in $R$ are fixed points of $T$.

The next result is useful for determining basins of attraction of fixed points of competitive maps.

Theorem 1.8. Assume the hypotheses of Theorem 1.5, and let $\mathcal{C}$ be the curve whose existence is guaranteed by Theorem 1.5. If the endpoints of $\mathcal{C}$ belong to $\partial \mathcal{R}$, then $\mathcal{C}$ separates $\mathcal{R}$ into two connected components, namely

$$
\mathcal{W}_{-}:=\left\{x \in \mathcal{R} \backslash \mathcal{C}: \exists y \in \mathcal{C} \text { with } x \varliminf_{s e} y\right\}, \quad \mathcal{W}_{+}:=\left\{x \in \mathcal{R} \backslash \mathcal{C}: \exists y \in \mathcal{C} \text { with } y \coprod_{s e} x\right\},
$$

such that the following statements are true.

(i) $\mathcal{W}_{-}$is invariant, and $\operatorname{dist}\left(T^{n}(x), Q_{2}(\bar{x})\right) \rightarrow 0$ as $n \rightarrow \infty$ for every $x \in \mathcal{W}_{-}$.

(ii) $\mathcal{W}_{+}$is invariant, and $\operatorname{dist}\left(T^{n}(x), Q_{4}(\bar{x})\right) \rightarrow 0$ as $n \rightarrow \infty$ for every $x \in \mathcal{W}_{+}$.

If, in addition, $\bar{x}$ is an interior point of $\mathcal{R}$ and $T$ is $C^{2}$ and strongly competitive in a neighborhood of $\bar{x}$, then $T$ has no periodic points in the boundary of $Q_{1}(\bar{x}) \cup Q_{3}(\bar{x})$ except for $\bar{x}$, and the following statements are true.

(iii) For every $x \in \mathcal{W}^{-}$, there exists $n_{0} \in \mathbb{N}$ such that $T^{n}(x) \in \operatorname{int} \mathcal{Q}_{2}(\bar{x})$ for $n \geq n_{0}$.

(iv) For every $x \in \mathcal{W}_{+}$, there exists $n_{0} \in \mathbb{N}$ such that $T^{n}(x) \in \operatorname{int} \mathcal{Q}_{4}(\bar{x})$ for $n \geq n_{0}$.

\section{Some Basic Facts}

In this section we give some basic facts about the nonexistence of period-two solutions, local injectivity of map $T$ at the equilibrium point and $(\mathrm{O}+)$ condition. 
Advances in Difference Equations

\subsection{Equilibrium Points}

The equilibrium points $(\bar{x}, \bar{y})$ of system (1.1) satisfy

$$
\begin{aligned}
& \bar{x}=\frac{\alpha_{1}+\beta_{1} \bar{x}}{\bar{y}}, \\
& \bar{y}=\frac{\alpha_{2}+\gamma_{2} \bar{y}}{A_{2}+\bar{x}}
\end{aligned}
$$

First equation of System (2.1) gives

$$
\bar{y}=\frac{\alpha_{1}}{\bar{x}}+\beta_{1}
$$

Second equation of System (2.1) gives

$$
\bar{y}\left(A_{2}+\bar{x}\right)=\alpha_{2}+\gamma_{2} \bar{y}
$$

Now, using (2.2), we obtain

$$
\frac{\left(\alpha_{1}+\beta_{1} \bar{x}\right)\left(A_{2}+\bar{x}\right)}{\bar{x}}=\alpha_{2}+\gamma_{2} \frac{\alpha_{1}+\beta_{1} \bar{x}}{\bar{x}} .
$$

This implies

$$
\left(\alpha_{1}+\beta_{1} \bar{x}\right)\left(A_{2}+\bar{x}\right)=\alpha_{2} \bar{x}+\gamma_{2} \alpha_{1}+\beta_{1} \gamma_{2} \bar{x}
$$

which is equivalent to

$$
\beta_{1} \bar{x}^{2}+\bar{x}\left[\left(\alpha_{1}-\alpha_{2}\right)+\beta_{1}\left(A_{2}-\gamma_{2}\right)\right]+\alpha_{1}\left(A_{2}-\gamma_{2}\right)=0 .
$$

Solutions of (2.6) are

$$
\begin{aligned}
& \bar{x}_{1}=\frac{-\left[\left(\alpha_{1}-\alpha_{2}\right)+\beta_{1}\left(A_{2}-\gamma_{2}\right)\right]+\sqrt{\left[\left(\alpha_{1}-\alpha_{2}\right)+\beta_{1}\left(A_{2}-\gamma_{2}\right)\right]^{2}-4 \alpha_{1} \beta_{1}\left(A_{2}-\gamma_{2}\right)}}{2 \beta_{1}}, \\
& \bar{x}_{2}=\frac{-\left[\left(\alpha_{1}-\alpha_{2}\right)+\beta_{1}\left(A_{2}-\gamma_{2}\right)\right]-\sqrt{\left[\left(\alpha_{1}-\alpha_{2}\right)+\beta_{1}\left(A_{2}-\gamma_{2}\right)\right]^{2}-4 \alpha_{1} \beta_{1}\left(A_{2}-\gamma_{2}\right)}}{2 \beta_{1}} .
\end{aligned}
$$


Table 1

\begin{tabular}{ll}
\hline$E_{1}$ & $A_{2}<\gamma_{2}$, \\
\hline$E_{1}, E_{2}$ & $A_{2}>\gamma_{2},\left[\left(\alpha_{1}-\alpha_{2}\right)+\beta_{1}\left(A_{2}-\gamma_{2}\right)\right]^{2}-4 \alpha_{1} \beta_{1}\left(A_{2}-\gamma_{2}\right)>0, \beta_{1}\left(A_{2}-\gamma_{2}\right)<\alpha_{2}-\alpha_{1}$ \\
$E_{1} \equiv E_{2}$ & $A_{2}>\gamma_{2},\left[\left(\alpha_{1}-\alpha_{2}\right)+\beta_{1}\left(A_{2}-\gamma_{2}\right)\right]^{2}-4 \alpha_{1} \beta_{1}\left(A_{2}-\gamma_{2}\right)=0, \beta_{1}\left(A_{2}-\gamma_{2}\right)<\alpha_{2}-\alpha_{1}$ \\
$E$ & $A_{2}=\gamma_{2}, \alpha_{2}>\alpha_{1}$ \\
No equilibrium & $A_{2}>\gamma_{2},\left[\left(\alpha_{1}-\alpha_{2}\right)+\beta_{1}\left(A_{2}-\gamma_{2}\right)\right]^{2}-4 \alpha_{1} \beta_{1}\left(A_{2}-\gamma_{2}\right)<0$ \\
No equilibrium & $A_{2}>\gamma_{2},\left[\left(\alpha_{1}-\alpha_{2}\right)+\beta_{1}\left(A_{2}-\gamma_{2}\right)\right]^{2}-4 \alpha_{1} \beta_{1}\left(A_{2}-\gamma_{2}\right) \geq 0, \beta_{1}\left(A_{2}-\gamma_{2}\right)>\alpha_{2}-\alpha_{1}$ \\
No equilibrium & $A_{2}=\gamma_{2}, \alpha_{2} \leq \alpha_{1}$ \\
\hline
\end{tabular}

Now, (2.2) gives

$$
\begin{aligned}
& \bar{y}_{1}=\frac{\left(\alpha_{1}-\alpha_{2}\right)-\beta_{1}\left(A_{2}-\gamma_{2}\right)+\sqrt{\left[\left(\alpha_{1}-\alpha_{2}\right)+\beta_{1}\left(A_{2}-\gamma_{2}\right)\right]^{2}-4 \alpha_{1} \beta_{1}\left(A_{2}-\gamma_{2}\right)}}{-2\left(A_{2}-\gamma_{2}\right)}, \\
& \bar{y}_{2}=\frac{\left(\alpha_{1}-\alpha_{2}\right)-\beta_{1}\left(A_{2}-\gamma_{2}\right)-\sqrt{\left[\left(\alpha_{1}-\alpha_{2}\right)+\beta_{1}\left(A_{2}-\gamma_{2}\right)\right]^{2}-4 \alpha_{1} \beta_{1}\left(A_{2}-\gamma_{2}\right)}}{-2\left(A_{2}-\gamma_{2}\right)} .
\end{aligned}
$$

The equilibrium points are:

$$
\begin{aligned}
& E_{1}=\left(\bar{x}_{1}, \bar{y}_{1}\right), \\
& E_{1}=\left(\bar{x}_{2}, \bar{y}_{2}\right),
\end{aligned}
$$

where $\bar{x}_{1}, \bar{y}_{1}, \bar{x}_{2}, \bar{y}_{2}$ are given by the above relations.

Note that

$$
\overline{x y} \neq \beta_{1} \gamma_{2} .
$$

The discriminant of (2.6) is given by

$$
D=\left[\left(\alpha_{1}-\alpha_{2}\right)+\beta_{1}\left(A_{2}-\gamma_{2}\right)\right]^{2}-4 \alpha_{1} \beta_{1}\left(A_{2}-\gamma_{2}\right) .
$$

The criteria for the existence of equilibrium points are summarized in Table 1 where

$$
E=\left(\frac{\alpha_{2}-\alpha_{1}}{\beta_{1}}, \frac{\beta_{1} \alpha_{2}}{\alpha_{2}-\alpha_{1}}\right) .
$$

\subsection{Condition $(O+)$ and Period-Two Solution}

In this section we prove three lemmas.

Lemma 2.1. System (1.1) satisfies either $(\mathrm{O}+)$ or $\left(\mathrm{O}_{-}\right)$. Consequently, the second iterate of every solution is eventually monotone. 
Proof. The map $T$ associated to system (1.1) is given by

$$
T(x, y)=\left(\frac{\alpha_{1}+\beta_{1} x}{y}, \frac{\alpha_{2}+\gamma_{2} y}{A_{2}+x}\right) .
$$

Assume

$$
T\left(x_{1}, y_{1}\right) \varliminf_{\mathrm{ne}} T\left(x_{2}, y_{2}\right),
$$

then we have

$$
\begin{aligned}
& \frac{\alpha_{1}+\beta_{1} x_{1}}{y_{1}} \leq \frac{\alpha_{1}+\beta_{1} x_{2}}{y_{2}} \\
& \frac{\alpha_{2}+\gamma_{2} y_{1}}{A_{2}+x_{1}} \leq \frac{\alpha_{2}+\gamma_{2} y_{2}}{A_{2}+x_{2}} .
\end{aligned}
$$

Equations (2.15) and (2.16) are equivalent, respectively, to

$$
\begin{gathered}
\alpha_{1}\left(y_{2}-y_{1}\right)+\beta_{1}\left(x_{1} y_{2}-x_{2} y_{1}\right) \leq 0 \\
\alpha_{2}\left(x_{2}-x_{1}\right)+A_{2} \gamma_{2}\left(y_{1}-y_{2}\right)+\gamma_{2}\left(x_{2} y_{1}-x_{1} y_{2}\right) \leq 0 .
\end{gathered}
$$

Now, using (2.17) and (2.18), we have the following:

$$
\begin{gathered}
\text { If } y_{2} \geq y_{1} \Longrightarrow x_{1} y_{2}<x_{2} y_{1} \Longrightarrow x_{1}<x_{2} \Longrightarrow\left(x_{1}, y_{1}\right) \leq_{\mathrm{ne}}\left(x_{2}, y_{2}\right) \\
\text { If } y_{2}<y_{1} \Longrightarrow x_{2}<x_{1} \text { and/or } x_{2} y_{1}-x_{1} y_{2}<0 \Longrightarrow x_{2}<x_{1} \Longrightarrow\left(x_{2}, y_{2}\right) \varliminf_{\mathrm{ne}}\left(x_{1}, y_{1}\right) \text {. }
\end{gathered}
$$

Lemma 2.2. System (1.1) has no minimal period-two solution.

Proof. Set

$$
T(x, y)=\left(\frac{\alpha_{1}+\beta_{1} x}{y}, \frac{\alpha_{2}+\gamma_{2} y}{A_{2}+x}\right)
$$

Then

$$
\begin{aligned}
T(T(x, y)) & =T\left(\frac{\alpha_{1}+\beta_{1} x}{y}, \frac{\alpha_{2}+\gamma_{2} y}{A_{2}+x}\right) \\
& =\left(\frac{\left(x+A_{2}\right)\left(\alpha_{1}+\left(\beta_{1}\left(\alpha_{1}+x \beta_{1}\right)\right) / y\right)}{\alpha_{2}+y \gamma_{2}}, \frac{y\left(\alpha_{2}+\left(\gamma_{2}\left(\alpha_{2}+y \gamma_{2}\right)\right) / x+A_{2}\right)}{y A_{2}+\alpha_{1}+x \beta_{1}}\right) .
\end{aligned}
$$


Period-two solution satisfies

$$
\begin{aligned}
& \frac{\left(x+A_{2}\right)\left(\alpha_{1}+\left(\beta_{1}\left(\alpha_{1}+x \beta_{1}\right)\right) / y\right)}{\alpha_{2}+y \gamma_{2}}-x=0, \\
& \frac{y\left(\alpha_{2}+\left(\gamma_{2}\left(\alpha_{2}+y \gamma_{2}\right)\right) /\left(x+A_{2}\right)\right)}{y A_{2}+\alpha_{1}+x \beta_{1}}-y=0 .
\end{aligned}
$$

We show that this system has no other positive solutions except equilibrium points.

Equations (2.22) and (2.23) are equivalent, respectively, to

$$
\begin{gathered}
\frac{x y \alpha_{1}+y A_{2} \alpha_{1}-x y \alpha_{2}+x \alpha_{1} \beta_{1}+A_{2} \alpha_{1} \beta_{1}+x^{2} \beta_{1}^{2}+x A_{2} \beta_{1}^{2}-x y^{2} \gamma_{2}}{y\left(\alpha_{2}+y \gamma_{2}\right)}=0 \\
\frac{-x y^{2} A_{2}-y^{2} A_{2}^{2}-x y \alpha_{1}-y A_{2} \alpha_{1}+x y \alpha_{2}+y A_{2} \alpha_{2}-x^{2} y \beta_{1}-x y A_{2} \beta_{1}+y \alpha_{2} \gamma_{2}+y^{2} \gamma_{2}^{2}}{\left(x+A_{2}\right)\left(y A_{2}+\alpha_{1}+x \beta_{1}\right)}=0
\end{gathered}
$$

Equation (2.24) implies

$$
y A_{2} \alpha_{1}+x y\left(\alpha_{1}-\alpha_{2}\right)+A_{2} \alpha_{1} \beta_{1}+x^{2} \beta_{1}^{2}+x \beta_{1}\left(\alpha_{1}+A_{2} \beta_{1}\right)-x y^{2} \gamma_{2}=0
$$

Equation (2.25) implies

$$
-x y^{2} A_{2}-x^{2} y \beta_{1}+x y\left(-\alpha_{1}+\alpha_{2}-A_{2} \beta_{1}\right)+y\left(A_{2}\left(-\alpha_{1}+\alpha_{2}\right)+\alpha_{2} \gamma_{2}\right)+y^{2}\left(-A_{2}^{2}+\gamma_{2}^{2}\right)=0 .
$$

Using (2.26), we have

$$
x^{2}=\frac{-y A_{2} \alpha_{1}-x y\left(\alpha_{1}-\alpha_{2}\right)-A_{2} \alpha_{1} \beta_{1}-x \beta_{1}\left(\alpha_{1}+A_{2} \beta_{1}\right)+x y^{2} \gamma_{2}}{\beta_{1}^{2}} .
$$

Putting (2.28) into (2.27), we have

$$
y\left(y\left(x+A_{2}\right) \alpha_{1}+y\left(-A_{2}\left(x+A_{2}\right) \beta_{1}-x y \gamma_{2}+\beta_{1} \gamma_{2}^{2}\right)+\alpha_{2}\left(-x y+\beta_{1}\left(x+A_{2}+\gamma_{2}\right)\right)\right)=0
$$

This is equivalent to

$$
x=\frac{-y A_{2}^{2} \beta_{1}+A_{2}\left(y \alpha_{1}+\alpha_{2} \beta_{1}\right)+\beta_{1} \gamma_{2}\left(\alpha_{2}+y \gamma_{2}\right)}{-y \alpha_{1}+\alpha_{2}\left(y-\beta_{1}\right)+y\left(A_{2} \beta_{1}+y \gamma_{2}\right)} .
$$


Putting (2.30) into (2.24), we obtain

$$
\beta_{1}\left(\alpha_{2}+y \gamma_{2}\right)\left(\alpha_{2} \beta_{1}+y^{2}\left(A_{2}-\gamma_{2}\right)+y\left(\alpha_{1}-\alpha_{2}+\beta_{1}\left(-A_{2}+\gamma_{2}\right)\right)\right)=0
$$

or

$y^{2} \gamma_{2}\left(A_{2}+\gamma_{2}\right)+\beta_{1} \gamma_{2}\left(-\alpha_{1}+\beta_{1}\left(A_{2}+\gamma_{2}\right)\right)+y\left(A_{2}+\gamma_{2}\right)\left(-\alpha_{1}+\alpha_{2}+\beta_{1}\left(A_{2}+\gamma_{2}\right)\right)=0$.

From (2.31), we obtain fixed points. In the sequel, we consider (2.32).

Discriminant of (2.32) is given by

$$
\Delta:=\left(A_{2}+\gamma_{2}\right)\left(-4 \beta_{1} \gamma_{2}^{2}\left(-\alpha_{1}+\beta_{1}\left(A_{2}+\gamma_{2}\right)\right)+\left(A_{2}+\gamma_{2}\right)\left(-\alpha_{1}+\alpha_{2}+\beta_{1}\left(A_{2}+\gamma_{2}\right)\right)^{2}\right) .
$$

Real solutions of (2.32) exist if and only if $\Delta \geq 0$. The solutions are given by

$$
\begin{aligned}
& y_{1}=\frac{\left(-A_{2}-\gamma_{2}\right)\left(-\alpha_{1}+\alpha_{2}+\beta_{1}\left(A_{2}+\gamma_{2}\right)\right)-\sqrt{\Delta}}{2 \gamma_{2}\left(A_{2}+\gamma_{2}\right)}, \\
& y_{2}=\frac{\left(-A_{2}-\gamma_{2}\right)\left(-\alpha_{1}+\alpha_{2}+\beta_{1}\left(A_{2}+\gamma_{2}\right)\right)+\sqrt{\Delta}}{2 \gamma_{2}\left(A_{2}+\gamma_{2}\right)} .
\end{aligned}
$$

Using (2.30), we have

$$
\begin{aligned}
& x_{1}=\frac{\left(-\alpha_{1}+\alpha_{2}+\beta_{1}\left(A_{2}-\gamma_{2}\right)\right)\left(A_{2}+\gamma_{2}\right)+\sqrt{\Delta}}{2 \beta_{1} \gamma_{2}}, \\
& x_{2}=\frac{\left(-\alpha_{1}+\alpha_{2}+\beta_{1}\left(A_{2}-\gamma_{2}\right)\right)\left(A_{2}+\gamma_{2}\right)-\sqrt{\Delta}}{2 \beta_{1} \gamma_{2}} .
\end{aligned}
$$

Claim. Assume $\Delta \geq 0$. Then

(i) for all values of parameters, $y_{1}<0$;

(ii) for all values of parameters, $x_{2}<0$.

Proof. (1) Assume $\left(-\alpha_{1}+\alpha_{2}+\beta_{1}\left(A_{2}+\gamma_{2}\right)\right)>0$. Then it is obvious that the claim $y_{1}<0$ is true. Now, assume $\left(-\alpha_{1}+\alpha_{2}+\beta_{1}\left(A_{2}+\gamma_{2}\right)\right) \leq 0$. Then $y_{1}<0$ if and only if

$$
\Delta-\left(A_{2}+\gamma_{2}\right)^{2}\left(-\alpha_{1}+\alpha_{2}+\beta_{1}\left(A_{2}+\gamma_{2}\right)\right)^{2}>0,
$$

which is equivalent to

$$
-4 \beta_{1} \gamma_{2}^{2}\left(A_{2}+\gamma_{2}\right)\left(-\alpha_{1}+\beta_{1}\left(A_{2}+\gamma_{2}\right)\right)>0
$$


This is true since

$$
\left(-\alpha_{1}+\beta_{1}\left(A_{2}+\gamma_{2}\right)\right)<\left(-\alpha_{1}+\alpha_{2}+\beta_{1}\left(A_{2}+\gamma_{2}\right)\right) \leq 0
$$

(2) Assume $\left(-\alpha_{1}+\alpha_{2}+\beta_{1}\left(A_{2}-\gamma_{2}\right)\right)<0$. Then it is obvious that $x_{2}<0$. Now, assume

$$
\left(-\alpha_{1}+\alpha_{2}+\beta_{1}\left(A_{2}-\gamma_{2}\right)\right) \geq 0
$$

Then $x_{2}<0$ if and only if

$$
\Delta-\left(-\alpha_{1}+\alpha_{2}+\beta_{1}\left(A_{2}-\gamma_{2}\right)\right)^{2}\left(A_{2}+\gamma_{2}\right)^{2}>0 .
$$

This is equivalent to

$$
4 \beta_{1} \gamma_{2}\left(A_{2}+\gamma_{2}\right)\left(A_{2}\left(-\alpha_{1}+\alpha_{2}+A_{2} \beta_{1}\right)+\alpha_{2} \gamma_{2}-\beta_{1} \gamma_{2}^{2}\right)>0
$$

Using (2.39), we have

$$
\left(A_{2}\left(-\alpha_{1}+\alpha_{2}+A_{2} \beta_{1}\right)+\alpha_{2} \gamma_{2}-\beta_{1} \gamma_{2}^{2}\right) \geq A_{2} \gamma_{2} \beta_{1}+\alpha_{2} \gamma_{2}-\beta_{1} \gamma_{2}^{2} \geq \alpha_{1} \gamma_{2}>0,
$$

which implies that the inequality (2.41) is true.

Now, the proof of the Lemma 2.2 follows from the Claim 2.2.

Lemma 2.3. The map $T$ associated to System (1.1) satisfies the following:

$$
T(x, y)=(\bar{x}, \bar{y}) \quad \text { only for }(x, y)=(\bar{x}, \bar{y}) \text {. }
$$

Proof. By using (2.1), we have

$$
\begin{gathered}
T(x, y)=(\bar{x}, \bar{y}), \\
\Longleftrightarrow \frac{\alpha_{1}+\beta_{1} x}{y}=\frac{\alpha_{1}+\beta_{1} \bar{x}}{\bar{y}}, \\
\frac{\alpha_{2}+\gamma_{2} y}{A_{2}+x}=\frac{\alpha_{2}+\gamma_{2} \bar{y}}{A_{2}+\bar{x}} .
\end{gathered}
$$

First equation implies

$$
\alpha_{1}(\bar{y}-y)+\beta_{1}(x \bar{y}-\bar{x} y)=0
$$

Second equation implies

$$
\alpha_{2}(\bar{x}-x)+\gamma_{2} A_{2}(y-\bar{y})=\gamma_{2}(x \bar{y}-y \bar{x}) .
$$


Advances in Difference Equations

Note the following

$$
x \bar{y}-\bar{x} y=(x-\bar{x}) \bar{y}+\bar{x}(\bar{y}-y) .
$$

Using (2.47), Equations (2.45) and (2.46), respectively, become

$$
\begin{gathered}
\beta_{1} \bar{y}(x-\bar{x})+\left(\alpha_{1}+\beta_{1} \bar{x}\right)(\bar{y}-y)=0, \\
\left(\alpha_{2}+\gamma_{2} \bar{y}\right)(x-\bar{x})+\gamma_{2}\left(A_{2}+\bar{x}\right)(\bar{y}-y)=0 .
\end{gathered}
$$

Note that System (2.48) is linear homogeneous system in $x-\bar{x}$ and $\bar{y}-y$. The determinant of System (2.48) is given by

$$
\left|\begin{array}{cc}
\beta_{1} \bar{y} & \alpha_{1}+\beta_{1} \bar{y} \\
\alpha_{2}+\gamma_{2} \bar{y} & \gamma_{2}\left(A_{2}+\bar{x}\right)
\end{array}\right|
$$

Using (2.1), the determinant of System (2.48) becomes

$$
\left|\begin{array}{cc}
\beta_{1} \bar{y} & \overline{x y} \\
\bar{y}\left(A_{2}+\bar{x}\right) & \gamma_{2}\left(A_{2}+\bar{x}\right)
\end{array}\right|=\bar{y}\left(A_{2}+\bar{x}\right)\left(\beta_{1} \gamma_{2}-\overline{x y}\right) \neq 0 .
$$

This implies that System (2.48) has only trivial solution, that is

$$
x=\bar{x}, \quad y=\bar{y} .
$$

\section{Linearized Stability Analysis}

The Jacobian matrix of the map $T$ has the following form:

$$
\mathrm{J}_{T}=\left(\begin{array}{cc}
\frac{\beta_{1}}{y} & -\frac{\alpha_{1}+\beta_{1} x}{y^{2}} \\
-\frac{\alpha_{2}+\gamma_{2} y}{\left(A_{2}+x\right)^{2}} & \frac{\gamma_{2}}{A_{2}+x}
\end{array}\right) .
$$

The value of the Jacobian matrix of $T$ at the equilibrium point is

$$
\mathrm{J}_{T}(\bar{x}, \bar{y})=\left(\begin{array}{cc}
\frac{\beta_{1}}{\bar{y}} & -\frac{\bar{x}}{\bar{y}} \\
-\bar{y} & \frac{\gamma_{2}}{A_{2}+\bar{x}}
\end{array}\right) .
$$


The determinant of (3.2) is given by

$$
\operatorname{det} \mathrm{J}_{T}(\bar{x}, \bar{y})=\frac{\beta_{1} \gamma_{2}-\overline{x y}}{\bar{y}\left(A_{2}+\bar{x}\right)}
$$

The trace of (3.2) is

$$
\operatorname{Tr} \mathrm{J}_{T}(\bar{x}, \bar{y})=\frac{\beta_{1}}{\bar{y}}+\frac{\gamma_{2}}{A_{2}+\bar{x}}
$$

The characteristic equation has the form

$$
\lambda^{2}-\lambda\left(\frac{\beta_{1}}{\bar{y}}+\frac{\gamma_{2}}{A_{2}+\bar{x}}\right)+\frac{\beta_{1} \gamma_{2}-\overline{x y}}{\bar{y}\left(A_{2}+\bar{x}\right)}=0
$$

Theorem 3.1. Assume that $A_{2}<\gamma_{2}$. Then there exists a unique positive equilibrium $E_{1}$ which is a saddle point, and the following statements hold.

(a) If $\beta_{1}\left(\gamma_{2}+A_{2}\right)<\alpha_{1}+\alpha_{2}$, then $\lambda_{1} \in(1,+\infty)$ and $\lambda_{2} \in(-1,0)$.

(b) If $\beta_{1}\left(\gamma_{2}+A_{2}\right)>\alpha_{1}+\alpha_{2}, \beta_{1} A_{2}<\alpha_{2}<\beta_{1} \gamma_{2}$ and $\alpha_{1}\left(\beta_{1} \gamma_{2}-\alpha_{2}\right)<\beta_{1} \gamma_{2}\left(\alpha_{2}-\beta_{1} A_{2}\right)$, then $\lambda_{1} \in(1,+\infty)$ and $\lambda_{2} \in(0,1)$.

(c) If $\beta_{1}\left(\gamma_{2}+A_{2}\right)>\alpha_{1}+\alpha_{2}, \alpha_{2}>\beta_{1} \gamma_{2}$ and $\alpha_{1}\left(\alpha_{2}-\beta_{1} \gamma_{2}\right)>\beta_{1} \gamma_{2}\left(\alpha_{2}-\beta_{1} A_{2}\right)$, then $\lambda_{1} \in(1,+\infty)$ and $\lambda_{2} \in(0,1)$.

(d) If $\beta_{1}\left(\gamma_{2}+A_{2}\right)>\alpha_{1}+\alpha_{2}, \alpha_{2}>\beta_{1} \gamma_{2}$ and $\alpha_{1}\left(\alpha_{2}-\beta_{1} \gamma_{2}\right)<\beta_{1} \gamma_{2}\left(\alpha_{2}-\beta_{1} A_{2}\right)$, then $\lambda_{1} \in(1,+\infty)$ and $\lambda_{2} \in(-1,0)$.

Proof. The equilibrium is a saddle point if and only if the following conditions are satisfied:

$$
\left|\operatorname{Tr} \mathrm{J}_{T}(\bar{x}, \bar{y})\right|>\left|1+\operatorname{det} \mathrm{J}_{T}(\bar{x}, \bar{y})\right|, \quad \operatorname{Tr}^{2} \mathrm{~J}_{T}(\bar{x}, \bar{y})-4 \operatorname{det} \mathrm{J}_{T}(\bar{x}, \bar{y})>0
$$

The first condition is equivalent to

$$
\frac{\beta_{1}}{\bar{y}}+\frac{\gamma_{2}}{A_{2}+\bar{x}}>1+\frac{\beta_{1} \gamma_{2}-\overline{x y}}{\bar{y}\left(A_{2}+\bar{x}\right)}
$$

This implies the following:

$$
\begin{aligned}
\beta_{1}\left(A_{2}+\bar{x}\right)+\gamma_{2} \bar{y}>\bar{y}\left(A_{2}+\bar{x}\right)+\beta_{1} \gamma_{2}-\overline{x y} \\
\quad \Longleftrightarrow\left(A_{2}+\bar{x}\right)\left(\beta_{1}-\bar{y}\right)+\gamma_{2}\left(\bar{y}-\beta_{1}\right)>-\overline{x y} \\
\Longleftrightarrow\left(\bar{y}-\beta_{1}\right)\left(A_{2}-\gamma_{2}+\bar{x}\right)<\overline{x y} .
\end{aligned}
$$


Notice the following:

$$
\begin{aligned}
\bar{y}_{1}-\beta_{1} & =\frac{\left(\alpha_{1}-\alpha_{2}\right)-\beta_{1}\left(A_{2}-\gamma_{2}\right)+\sqrt{\left[\left(\alpha_{1}-\alpha_{2}\right)+\beta_{1}\left(A_{2}-\gamma_{2}\right)\right]^{2}-4 \alpha_{1} \beta_{1}\left(A_{2}-\gamma_{2}\right)}}{-2\left(A_{2}-\gamma_{2}\right)}-\beta_{1} \\
& =\frac{\left(\alpha_{1}-\alpha_{2}\right)+\beta_{1}\left(A_{2}-\gamma_{2}\right)+\sqrt{\left[\left(\alpha_{1}-\alpha_{2}\right)+\beta_{1}\left(A_{2}-\gamma_{2}\right)\right]^{2}-4 \alpha_{1} \beta_{1}\left(A_{2}-\gamma_{2}\right)}}{-2\left(A_{2}-\gamma_{2}\right)} \\
& =\frac{-\left[\left(\alpha_{1}-\alpha_{2}\right)+\beta_{1}\left(A_{2}-\gamma_{2}\right)\right]-\sqrt{\left[\left(\alpha_{1}-\alpha_{2}\right)+\beta_{1}\left(A_{2}-\gamma_{2}\right)\right]^{2}-4 \alpha_{1} \beta_{1}\left(A_{2}-\gamma_{2}\right)}}{2 \beta_{1}} \frac{2 \beta_{1}}{2\left(A_{2}-\gamma_{2}\right)} \\
& =\bar{x}_{2} \frac{\beta_{1}}{A_{2}-\gamma_{2}} .
\end{aligned}
$$

That is,

$$
\bar{y}_{1}-\beta_{1}=\bar{x}_{2} \frac{\beta_{1}}{A_{2}-\gamma_{2}} .
$$

Similarly,

$$
\begin{aligned}
A_{2}-\gamma_{2}+\bar{x}_{1}= & A_{2}-\gamma_{2} \\
& +\frac{-\left[\left(\alpha_{1}-\alpha_{2}\right)+\beta_{1}\left(A_{2}-\gamma_{2}\right)\right]+\sqrt{\left[\left(\alpha_{1}-\alpha_{2}\right)+\beta_{1}\left(A_{2}-\gamma_{2}\right)\right]^{2}-4 \alpha_{1} \beta_{1}\left(A_{2}-\gamma_{2}\right)}}{2 \beta_{1}} \\
= & \frac{\beta_{1}\left(A_{2}-\gamma_{2}\right)-\left(\alpha_{1}-\alpha_{2}\right)+\sqrt{\left[\left(\alpha_{1}-\alpha_{2}\right)+\beta_{1}\left(A_{2}-\gamma_{2}\right)\right]^{2}-4 \alpha_{1} \beta_{1}\left(A_{2}-\gamma_{2}\right)}}{2 \beta_{1}} \\
= & \frac{-\beta_{1}\left(A_{2}-\gamma_{2}\right)+\left(\alpha_{1}-\alpha_{2}\right)-\sqrt{\left[\left(\alpha_{1}-\alpha_{2}\right)+\beta_{1}\left(A_{2}-\gamma_{2}\right)\right]^{2}-4 \alpha_{1} \beta_{1}\left(A_{2}-\gamma_{2}\right)}}{-2 \beta_{1}} \\
= & \frac{\left(\alpha_{1}-\alpha_{2}\right)-\beta_{1}\left(A_{2}-\gamma_{2}\right)-\sqrt{\left[\left(\alpha_{1}-\alpha_{2}\right)+\beta_{1}\left(A_{2}-\gamma_{2}\right)\right]^{2}-4 \alpha_{1} \beta_{1}\left(A_{2}-\gamma_{2}\right)}}{-2\left(A_{2}-\gamma_{2}\right)} \frac{A_{2}-\gamma_{2}}{\beta_{1}} \\
= & \bar{y}_{2} \frac{A_{2}-\gamma_{2}}{\beta_{1}} .
\end{aligned}
$$

Now, we have

$$
\left(\bar{y}_{1}-\beta_{1}\right)\left(A_{2}-\gamma_{2}+\bar{x}_{1}\right)<\bar{x}_{1} \bar{y}_{1} \Longleftrightarrow \bar{x}_{2} \frac{\beta_{1}}{A_{2}-\gamma_{2}} \bar{y}_{2} \frac{A_{2}-\gamma_{2}}{\beta_{1}}<\bar{x}_{1} \bar{y}_{1} .
$$


This is equivalent to

$$
\bar{x}_{2} \bar{y}_{2}<\bar{x}_{1} \bar{y}_{1} \text {. }
$$

The last condition is equivalent to

$$
\bar{x}_{2} \bar{y}_{2}-\bar{x}_{1} \bar{y}_{1}<0 \Longleftrightarrow-\left(\bar{y}_{2}\left(\bar{x}_{1}-\bar{x}_{2}\right)+\bar{x}_{1}\left(\bar{y}_{1}-\bar{y}_{2}\right)\right)<0
$$

which is true since $\bar{x}_{1}>\bar{x}_{2}$ and $\bar{y}_{1}>\bar{y}_{2}$.

The second condition is equivalent to

$$
\left(\frac{\beta_{1}}{\bar{y}}+\frac{\gamma_{2}}{A_{2}+\bar{x}}\right)^{2}-4 \frac{\beta_{1} \gamma_{2}}{\bar{y}\left(A_{2}+\bar{x}\right)}+4 \frac{\overline{x y}}{\bar{y}\left(A_{2}+\bar{x}\right)}>0
$$

This is equivalent to

$$
\left(\frac{\beta_{1}}{\bar{y}}-\frac{\gamma_{2}}{A_{2}+\bar{x}}\right)^{2}+4 \frac{\overline{x y}}{\bar{y}\left(A_{2}+\bar{x}\right)}>0
$$

establishing the proof of Theorem 3.1.

Since the map $T$ is strongly competitive, the Jacobian matrix (3.2) has two real and distinct eigenvalues, with the larger one in absolute value being positive.

From (3.5) at $E_{1}$, we have

$$
\begin{aligned}
\lambda_{1}+\lambda_{2} & =\frac{\beta_{1}}{\bar{y}_{1}}+\frac{\gamma_{2}}{A_{2}+\bar{x}_{1}}, \\
\lambda_{1} \lambda_{2} & =\frac{\beta_{1} \gamma_{2}-\bar{x}_{1} \bar{y}_{1}}{\bar{y}_{1}\left(A_{2}+\bar{x}_{1}\right)} .
\end{aligned}
$$

The first equation implies that either both eigenvalues are positive or the smaller one is negative.

Consider the numerator of the right-hand side of the second equation. We have

$$
\begin{aligned}
\beta_{1} \gamma_{2}-\bar{x}_{1} \bar{y}_{1} & =\beta_{1} \gamma_{2}-\frac{-\left(\alpha_{1}-\alpha_{2}\right)-\beta_{1}\left(A_{2}-\gamma_{2}\right)+\sqrt{D}}{2 \beta_{1}} \frac{\left(\alpha_{1}-\alpha_{2}\right)-\beta_{1}\left(A_{2}-\gamma_{2}\right)+\sqrt{D}}{-2\left(A_{2}-\gamma_{2}\right)} \\
& =\beta_{1} \gamma_{2}-\frac{\sqrt{D}+\left(\alpha_{1}+\alpha_{2}\right)-\beta_{1}\left(A_{2}-\gamma_{2}\right)}{2} \\
& =\frac{\beta_{1}\left(\gamma_{2}+A_{2}\right)-\left(\alpha_{1}+\alpha_{2}\right)-\sqrt{D}}{2},
\end{aligned}
$$

where $D=\left[\left(\alpha_{1}-\alpha_{2}\right)+\beta_{1}\left(A_{2}-\gamma_{2}\right)\right]^{2}-4 \alpha_{1} \beta_{1}\left(A_{2}-\gamma_{2}\right)$. 
(a) If $\beta_{1}\left(\gamma_{2}+A_{2}\right)<\alpha_{1}+\alpha_{2}$, then the smaller root is negative, that is, $\lambda_{2} \in(-1,0)$.

If $\beta_{1}\left(\gamma_{2}+A_{2}\right)>\left(\alpha_{1}+\alpha_{2}\right)$, then

$$
\begin{aligned}
\beta_{1}\left(\gamma_{2}+A_{2}\right)-\left(\alpha_{1}+\alpha_{2}\right)>\sqrt{D} \\
\quad \Longleftrightarrow \beta_{1}^{2}\left(\gamma_{2}+A_{2}\right)^{2}-2 \beta_{1}\left(\gamma_{2}+A_{2}\right)\left(\alpha_{1}+\alpha_{2}\right)+\left(\alpha_{1}+\alpha_{2}\right)^{2} \\
>\left(\alpha_{1}-\alpha_{2}\right)^{2}+2 \beta_{1}\left(\alpha_{1}-\alpha_{2}\right)\left(A_{2}-\gamma_{2}\right)+\beta_{1}^{2}\left(A_{2}-\gamma_{2}\right)^{2}-4 \alpha_{1} \beta_{1}\left(A_{2}-\gamma_{2}\right) \\
\quad \Longleftrightarrow \beta_{1} \gamma_{2}\left(\beta_{1} A_{2}-\alpha_{2}\right)+\alpha_{1}\left(\alpha_{2}-\beta_{1} \gamma_{2}\right)>0 .
\end{aligned}
$$

From the last inequality statements $(b),(c)$ and $(d)$ follow.

We now perform a similar analysis for the other cases in Table 1.

Theorem 3.2. Assume

$$
A_{2}>\gamma_{2}, \quad \beta_{1}\left(A_{2}-\gamma_{2}\right)<\alpha_{2}-\alpha_{1}, \quad\left[\left(\alpha_{1}-\alpha_{2}\right)+\beta_{1}\left(A_{2}-\gamma_{2}\right)\right]^{2}-4 \alpha_{1} \beta_{1}\left(A_{2}-\gamma_{2}\right)>0 .
$$

Then $E_{1}, E_{2}$ exist. $E_{1}$ is a saddle point; $E_{2}$ is a sink. For the eigenvalues of $E_{1}, \lambda_{1}\left(E_{1}\right) \in(1,+\infty)$ the following holds.

(a) If $\beta_{1} \gamma_{2}<\alpha_{2}<\beta_{1} A_{2}$, then $\lambda_{2} \in(0,1)$.

(b) If $\alpha_{2}>\beta_{1} A_{2}$ and $\alpha_{1}\left(\alpha_{2}-\beta_{1} \gamma_{2}\right)<\beta_{1} \gamma_{2}\left(\alpha_{2}-\beta_{1} A_{2}\right)$, then $\lambda_{2} \in(-1,0)$.

(c) If $\alpha_{2}<\beta_{1} \gamma_{2}$ and $\beta_{1} \gamma_{2}\left(\beta_{1} A_{2}-\alpha_{2}\right)>\alpha_{1}\left(\beta_{1} \gamma_{2}-\alpha_{2}\right)$, then $\lambda_{2} \in(0,1)$.

Proof. Note that if $\beta_{1} A_{2}-\alpha_{2}<0$ and $\alpha_{2}-\beta_{1} \gamma_{2}<0$, then $\alpha_{2}>\beta_{1} A_{2}$ and $\alpha_{2}<\beta_{1} \gamma_{2}$, which implies $A_{2}<\gamma_{2}$, which is a contradiction.

The equilibrium is a sink if the following condition is satisfied:

$$
\left|\operatorname{Tr} \mathrm{J}_{T}(\bar{x}, \bar{y})\right|<\left|1+\operatorname{det} \mathrm{J}_{T}(\bar{x}, \bar{y})\right|<2
$$

The condition $\left|\operatorname{Tr} \mathrm{J}_{T}(\bar{x}, \bar{y})\right|<\left|1+\operatorname{det} \mathrm{J}_{T}(\bar{x}, \bar{y})\right|$ is equivalent to

$$
\frac{\beta_{1}}{\bar{y}}+\frac{\gamma_{2}}{A_{2}+\bar{y}}<1+\frac{\beta_{1} \gamma_{2}}{\bar{y}\left(A_{2}+\bar{x}\right)}-\frac{\overline{x y}}{\bar{y}\left(A_{2}+\bar{x}\right)}
$$

This implies

$$
\begin{aligned}
\beta_{1}\left(A_{2}+\bar{x}\right)+\gamma_{2} \bar{y}<\bar{y}\left(A_{2}+\bar{x}\right)+\beta_{1} \gamma_{2}-\overline{x y} \\
\quad \Longleftrightarrow\left(A_{2}+\bar{x}\right)\left(\beta_{1}-\bar{y}\right)+\gamma_{2}\left(\bar{y}-\beta_{1}\right)<-\overline{x y} \\
\Longleftrightarrow\left(\bar{y}-\beta_{1}\right)\left(A_{2}-\gamma_{2}+\bar{x}\right)>\overline{x y} .
\end{aligned}
$$

Now, we prove that $E_{2}$ is a sink. 
We have to prove that

$$
\left(\bar{y}_{2}-\beta_{1}\right)\left(A_{2}-\gamma_{2}+\bar{x}_{2}\right)>\bar{x}_{2} \bar{y}_{2} .
$$

Notice the following:

$$
\begin{aligned}
\bar{y}_{2}-\beta_{1} & =\frac{\left(\alpha_{1}-\alpha_{2}\right)-\beta_{1}\left(A_{2}-\gamma_{2}\right)-\sqrt{\left[\left(\alpha_{1}-\alpha_{2}\right)+\beta_{1}\left(A_{2}-\gamma_{2}\right)\right]^{2}-4 \alpha_{1} \beta_{1}\left(A_{2}-\gamma_{2}\right)}}{-2\left(A_{2}-\gamma_{2}\right)}-\beta_{1} \\
& =\frac{\left(\alpha_{1}-\alpha_{2}\right)+\beta_{1}\left(A_{2}-\gamma_{2}\right)-\sqrt{\left[\left(\alpha_{1}-\alpha_{2}\right)+\beta_{1}\left(A_{2}-\gamma_{2}\right)\right]^{2}-4 \alpha_{1} \beta_{1}\left(A_{2}-\gamma_{2}\right)}}{-2\left(A_{2}-\gamma_{2}\right)} \\
& =\frac{-\left[\left(\alpha_{1}-\alpha_{2}\right)+\beta_{1}\left(A_{2}-\gamma_{2}\right)\right]+\sqrt{\left[\left(\alpha_{1}-\alpha_{2}\right)+\beta_{1}\left(A_{2}-\gamma_{2}\right)\right]^{2}-4 \alpha_{1} \beta_{1}\left(A_{2}-\gamma_{2}\right)}}{2\left(A_{2}-\gamma_{2}\right)} \\
& =\frac{-\left[\left(\alpha_{1}-\alpha_{2}\right)+\beta_{1}\left(A_{2}-\gamma_{2}\right)\right]+\sqrt{\left[\left(\alpha_{1}-\alpha_{2}\right)+\beta_{1}\left(A_{2}-\gamma_{2}\right)\right]^{2}-4 \alpha_{1} \beta_{1}\left(A_{2}-\gamma_{2}\right)}}{2\left(A_{2}-\gamma_{2}\right)} \frac{\beta_{1}}{A_{2}-\gamma_{2}} \\
& =\bar{x}_{1} \frac{\beta_{1}}{A_{2}-\gamma_{2}} .
\end{aligned}
$$

Similarly,

$$
\begin{aligned}
A_{2}-\gamma_{2}+\bar{x}_{2}= & A_{2}-\gamma_{2} \\
& +\frac{-\left[\left(\alpha_{1}-\alpha_{2}\right)+\beta_{1}\left(A_{2}-\gamma_{2}\right)\right]-\sqrt{\left[\left(\alpha_{1}-\alpha_{2}\right)+\beta_{1}\left(A_{2}-\gamma_{2}\right)\right]^{2}-4 \alpha_{1} \beta_{1}\left(A_{2}-\gamma_{2}\right)}}{2 \beta_{1}} \\
= & \frac{\left(\alpha_{1}-\alpha_{2}\right)-\beta_{1}\left(A_{2}-\gamma_{2}\right)+\sqrt{\left[\left(\alpha_{1}-\alpha_{2}\right)+\beta_{1}\left(A_{2}-\gamma_{2}\right)\right]^{2}-4 \alpha_{1} \beta_{1}\left(A_{2}-\gamma_{2}\right)}}{-2 \beta_{1}} \\
= & \frac{\left(\alpha_{1}-\alpha_{2}\right)-\beta_{1}\left(A_{2}-\gamma_{2}\right)+\sqrt{\left[\left(\alpha_{1}-\alpha_{2}\right)+\beta_{1}\left(A_{2}-\gamma_{2}\right)\right]^{2}-4 \alpha_{1} \beta_{1}\left(A_{2}-\gamma_{2}\right)}}{-2\left(A_{2}-\gamma_{2}\right)} \frac{A_{2}-\gamma_{2}}{\beta_{1}} \\
= & \bar{y}_{1} \frac{A_{2}-\gamma_{2}}{\beta_{1}} .
\end{aligned}
$$

Now, condition

$$
\left(\bar{y}_{2}-\beta_{1}\right)\left(A_{2}-\gamma_{2}+\bar{x}_{2}\right)>\bar{x}_{2} \bar{y}_{2}
$$


becomes

$$
\bar{x}_{1} \frac{\beta_{1}}{A_{2}-\gamma_{2}} \bar{y}_{1} \frac{A_{2}-\gamma_{2}}{\beta_{1}}>\bar{x}_{2} \bar{y}_{2}
$$

that is,

$$
\bar{x}_{1} \bar{y}_{1}>\bar{x}_{2} \bar{y}_{2}
$$

which is true. (see Theorem 3.1.)

Condition

$$
\left|1+\operatorname{det} \mathrm{J}_{T}(\bar{x}, \bar{y})\right|<2
$$

is equivalent to

$$
\frac{\beta_{1} \gamma_{2}}{\bar{y}\left(A_{2}+\bar{x}\right)}-\frac{\overline{x y}}{\bar{y}\left(A_{2}+\bar{x}\right)}<1
$$

This implies

$$
\beta_{1} \gamma_{2}-\overline{x y}<\bar{y}\left(A_{2}+\bar{x}\right) \Longleftrightarrow \beta_{1} \gamma_{1}-\bar{y} A_{2}<2 \overline{x y}
$$

We have to prove that

$$
\beta_{1} \gamma_{2}-\bar{y}_{2} A_{2}<2 \bar{x}_{2} \bar{y}_{2}
$$

Using (2.2), we have

$$
\beta_{1} \gamma_{2}-\left(\frac{\alpha_{1}}{\bar{x}_{2}}+\beta_{1}\right) A_{2}<2 \bar{x}_{2}\left(\frac{\alpha_{1}}{\bar{x}_{2}}+\beta_{1}\right) .
$$

This is equivalent to

$$
\beta_{1}\left(\gamma_{2}-A_{2}\right)<2 \alpha_{1}+2 \bar{x}_{2} \beta_{1}+\frac{\alpha_{1} A_{2}}{\bar{x}_{2}}
$$

which is always true since $A_{2}>\gamma_{2}$ and the left side is always negative, while the right side is always positive. 
Notice that conditions

$$
\begin{gathered}
\bar{x}_{1} \bar{y}_{1}>\bar{x}_{2} \bar{y}_{2}, \\
\left(\frac{\beta_{1}}{\bar{y}}-\frac{\gamma_{2}}{A_{2}+\bar{x}}\right)^{2}+4 \frac{\overline{x y}}{\bar{y}\left(A_{2}+\bar{x}\right)}>0,
\end{gathered}
$$

imply that $E_{1}$ is a saddle point.

From (3.5) at $E_{1}$, we have

$$
\begin{aligned}
\lambda_{1}+\lambda_{2} & =\frac{\beta_{1}}{\bar{y}_{1}}+\frac{\gamma_{2}}{A_{2}+\bar{x}_{1}}, \\
\lambda_{1} \lambda_{2} & =\frac{\beta_{1} \gamma_{2}-\bar{x}_{1} \bar{y}_{1}}{\bar{y}_{1}\left(A_{2}+\bar{x}_{1}\right)} .
\end{aligned}
$$

The first equation implies that either both eigenvalues are positive or the smaller one is negative.

Consider the numerator of the right-hand side of the second equation. We have

$$
\begin{aligned}
\beta_{1} \gamma_{2}-\bar{x}_{1} \bar{y}_{1} & =\beta_{1} \gamma_{2}-\frac{-\left(\alpha_{1}-\alpha_{2}\right)-\beta_{1}\left(A_{2}-\gamma_{2}\right)+\sqrt{D}}{2 \beta_{1}} \frac{\left(\alpha_{1}-\alpha_{2}\right)-\beta_{1}\left(A_{2}-\gamma_{2}\right)+\sqrt{D}}{-2\left(A_{2}-\gamma_{2}\right)} \\
& =\beta_{1} \gamma_{2}-\frac{\sqrt{D}+\left(\alpha_{1}+\alpha_{2}\right)-\beta_{1}\left(A_{2}-\gamma_{2}\right)}{2} \\
& =\frac{\beta_{1}\left(\gamma_{2}+A_{2}\right)-\left(\alpha_{1}+\alpha_{2}\right)-\sqrt{D}}{2} .
\end{aligned}
$$

We have

$$
\beta_{1} \gamma_{2}-\overline{x y}>0 \Longleftrightarrow \frac{\beta_{1}\left(\gamma_{2}+A_{2}\right)-\left(\alpha_{1}+\alpha_{2}\right)-\sqrt{D}}{2}>0
$$

Inequality

$$
\beta_{1}\left(\gamma_{2}+A_{2}\right)-\left(\alpha_{1}+\alpha_{2}\right)-\sqrt{D}>0
$$

is equivalent to

$$
\beta_{1} \gamma_{2}\left(\beta_{1} A_{2}-\alpha_{2}\right)+\alpha_{1}\left(\alpha_{2}-\beta_{1} \gamma_{2}\right)>0
$$

which is obvious if $\beta_{1} \gamma_{2}<\alpha_{2}<\beta_{1} A_{2}$. Then inequality (3.41) holds. This confirms (a). The other cases follow from (3.41). 
Theorem 3.3. Assume

$$
A_{2}>\gamma_{2}, \quad \beta_{1}\left(A_{2}-\gamma_{2}\right)<\alpha_{2}-\alpha_{1}, \quad\left[\left(\alpha_{1}-\alpha_{2}\right)+\beta_{1}\left(A_{2}-\gamma_{2}\right)\right]^{2}-4 \alpha_{1} \beta_{1}\left(A_{2}-\gamma_{2}\right)=0 .
$$

Then there exists a unique positive equilibrium point

$$
E_{1}=E_{2}=\left(\frac{\left(\alpha_{2}-\alpha_{1}\right)-\beta_{1}\left(A_{2}-\gamma_{2}\right)}{2 \beta_{1}}, \frac{\beta_{1}\left(A_{2}-\gamma_{2}\right)-\left(\alpha_{1}-\alpha_{2}\right)}{2\left(A_{2}-\gamma_{2}\right)}\right),
$$

which is non-hyperbolic. The following holds.

(a) If $\beta_{1}\left(A_{2}+\gamma_{2}\right)>\alpha_{1}+\alpha_{2}$, then $\lambda_{1}=1$ and $\lambda_{2} \in(0,1)$.

(b) If $\beta_{1}\left(A_{2}+\gamma_{2}\right)<\alpha_{1}+\alpha_{2}$, then $\lambda_{1}=1$ and $\lambda_{2} \in(-1,0)$.

Proof. Evaluating the Jacobian matrix (3.2) at equilibrium $E_{1}=E_{2}=\left(\left(\left(\alpha_{2}-\alpha_{1}\right)-\beta_{1}\left(A_{2}-\right.\right.\right.$ $\left.\left.\left.\gamma_{2}\right)\right) / 2 \beta_{1},\left(\beta_{1}\left(A_{2}-\gamma_{2}\right)-\left(\alpha_{1}-\alpha_{2}\right)\right) / 2\left(A_{2}-\gamma_{2}\right)\right)$, we have

$$
\begin{aligned}
& \mathrm{J}_{T}(\bar{x}, \bar{y}) \\
& \quad=\left(\begin{array}{cc}
\frac{\beta_{1}}{\left(\beta_{1}\left(A_{2}-\gamma_{2}\right)-\left(\alpha_{1}-\alpha_{2}\right)\right) / 2\left(A_{2}-\gamma_{2}\right)} & -\frac{\left(\left(\alpha_{2}-\alpha_{1}\right)-\beta_{1}\left(A_{2}-\gamma_{2}\right)\right) / 2 \beta_{1}}{\left(\beta_{1}\left(A_{2}-\gamma_{2}\right)-\left(\alpha_{1}-\alpha_{2}\right)\right) / 2\left(A_{2}-\gamma_{2}\right)} \\
-\frac{\left(\beta_{1}\left(A_{2}-\gamma_{2}\right)-\left(\alpha_{1}-\alpha_{2}\right)\right) / 2\left(A_{2}-\gamma_{2}\right)}{A_{2}+\left(\left(\left(\alpha_{2}-\alpha_{1}\right)-\beta_{1}\left(A_{2}-\gamma_{2}\right)\right) / 2 \beta_{1}\right)} & \frac{\gamma_{2}}{A_{2}+\left(\left(\left(\alpha_{2}-\alpha_{1}\right)-\beta_{1}\left(A_{2}-\gamma_{2}\right)\right) / 2 \beta_{1}\right)}
\end{array}\right)
\end{aligned}
$$

The characteristic equation of $\mathrm{J}_{T}(\bar{x}, \bar{y})$ is

$$
\begin{aligned}
\operatorname{det}\left(\mathrm{J}_{T}(\bar{x}, \bar{y})-\lambda I\right) & \\
= & \left(\begin{array}{cc}
\frac{\beta_{1}}{\left(\beta_{1}\left(A_{2}-\gamma_{2}\right)-\left(\alpha_{1}-\alpha_{2}\right)\right) / 2\left(A_{2}-\gamma_{2}\right)}-\lambda & -\frac{\left(\left(\alpha_{2}-\alpha_{1}\right)-\beta_{1}\left(A_{2}-\gamma_{2}\right)\right) / 2 \beta_{1}}{\left(\beta_{1}\left(A_{2}-\gamma_{2}\right)-\left(\alpha_{1}-\alpha_{2}\right)\right) / 2\left(A_{2}-\gamma_{2}\right)} \\
-\frac{\left(\beta_{1}\left(A_{2}-\gamma_{2}\right)-\left(\alpha_{1}-\alpha_{2}\right)\right) / 2\left(A_{2}-\gamma_{2}\right)}{A_{2}+\left(\left(\left(\alpha_{2}-\alpha_{1}\right)-\beta_{1}\left(A_{2}-\gamma_{2}\right)\right) / 2 \beta_{1}\right)} & \frac{\gamma_{2}}{A_{2}+\left(\left(\left(\alpha_{2}-\alpha_{1}\right)-\beta_{1}\left(A_{2}-\gamma_{2}\right)\right) / 2 \beta_{1}\right)}-\lambda
\end{array}\right) \\
= & 0,
\end{aligned}
$$

which is simplified to

$$
\begin{gathered}
\lambda^{2}-\lambda\left(\frac{\beta_{1}}{\left(\beta_{1}\left(A_{2}-\gamma_{2}\right)-\left(\alpha_{1}-\alpha_{2}\right)\right) / 2\left(A_{2}-\gamma_{2}\right)}+\frac{\gamma_{2}}{A_{2}+\left(\left(\left(\alpha_{2}-\alpha_{1}\right)-\beta_{1}\left(A_{2}-\gamma_{2}\right)\right) / 2 \beta_{1}\right)}\right) \\
+\frac{\beta_{1} \gamma_{2}-\left(\left(\left(\alpha_{2}-\alpha_{1}\right)-\beta_{1}\left(A_{2}-\gamma_{2}\right)\right) / 2 \beta_{1}\right)\left(\left(\beta_{1}\left(A_{2}-\gamma_{2}\right)-\left(\alpha_{1}-\alpha_{2}\right)\right) / 2\left(A_{2}-\gamma_{2}\right)\right)}{\left(\left(\beta_{1}\left(A_{2}-\gamma_{2}\right)-\left(\alpha_{1}-\alpha_{2}\right)\right) / 2\left(A_{2}-\gamma_{2}\right)\right)\left(A_{2}+\left(\left(\left(\alpha_{2}-\alpha_{1}\right)-\beta_{1}\left(A_{2}-\gamma_{2}\right)\right) / 2 \beta_{1}\right)\right)}=0 .
\end{gathered}
$$


Solutions of (3.46) are

$$
\lambda_{1}=1, \quad \lambda_{2}=\frac{-\alpha_{1}^{2}+2 \alpha_{1} \alpha_{2}-\alpha_{2}^{2}+A_{2}^{2} \beta_{1}^{2}+2 A_{2} \beta_{1}^{2} \gamma_{2}-3 \beta_{1}^{2} \gamma_{2}^{2}}{\alpha_{1}^{2}-2 \alpha_{1} \alpha_{2}+\alpha_{2}^{2}-2 A_{2} \alpha_{1} \beta_{1}+2 A_{2} \alpha_{2} \beta_{1}+A_{2}^{2} \beta_{1}^{2}-\beta_{1}^{2} \gamma_{2}^{2}}
$$

Note that $\lambda_{2}$ can be written in the following form:

$$
\lambda_{2}=\frac{-\left(\alpha_{1}-\alpha_{2}\right)^{2}+\beta_{1}^{2}\left(A_{2}^{2}-\gamma_{2}^{2}\right)+2 \beta_{1}^{2} \gamma_{2}\left(A_{2}-\gamma_{2}\right)}{\left(\alpha_{1}-\alpha_{2}\right)^{2}+2 A_{2} \beta_{1}\left(\alpha_{2}-\alpha_{1}\right)+\beta_{1}^{2}\left(A_{2}^{2}-\gamma_{2}^{2}\right)} \text {. }
$$

Note that $\left|\lambda_{2}\right|<1$.

The corresponding eigenvectors, respectively, are

$$
\left(-\frac{A_{2}-\gamma_{2}}{\beta_{1}}, 1\right), \quad\left(-\frac{\left(A_{2}-\gamma_{2}\right)\left(\alpha_{1}-\alpha_{2}+A_{2} \beta_{1}-\beta_{1} \gamma_{2}\right)\left(-\alpha_{1}+\alpha_{2}+A_{2} \beta_{1}+\beta_{1} \gamma_{2}\right)}{\beta_{1}\left(-\alpha_{1}+\alpha_{2}+A_{2} \beta_{1}-\beta_{1} \gamma_{2}\right)^{2}}, 1\right) .
$$

Note that the denominator of (3.48) is always positive.

Consider numerator of (3.48)

$$
-\left(\alpha_{1}-\alpha_{2}\right)^{2}+\beta_{1}^{2}\left(A_{2}^{2}-\gamma_{2}^{2}\right)+2 \beta_{1}^{2} \gamma_{2}\left(A_{2}-\gamma_{2}\right)
$$

From

$$
\left[\left(\alpha_{1}-\alpha_{2}\right)+\beta_{1}\left(A_{2}-\gamma_{2}\right)\right]^{2}-4 \alpha_{1} \beta_{1}\left(A_{2}-\gamma_{2}\right)=0,
$$

we have

$$
\left(\alpha_{1}-\alpha_{2}\right)^{2}=4 \alpha_{1} \beta_{1}\left(A_{2}-\gamma_{2}\right)-2 \beta_{1}\left(\alpha_{1}-\alpha_{2}\right)\left(A_{2}-\gamma_{2}\right)-\beta_{1}^{2}\left(A_{2}-\gamma_{2}\right)^{2} .
$$

Substituting $\left(\alpha_{1}-\alpha_{2}\right)^{2}$ from (3.52) in (3.50), we obtain

$$
\begin{aligned}
& -4 \alpha_{1} \beta_{1}\left(A_{2}-\gamma_{2}\right)+2 \beta_{1}\left(\alpha_{1}-\alpha_{2}\right)\left(A_{2}-\gamma_{2}\right)+\beta_{2}^{2}\left(A_{2}^{2}-\gamma_{2}^{2}\right)+2 \beta_{1}^{2} \gamma_{2}\left(A_{2}-\gamma_{2}\right) \\
& =2 \beta_{1}\left(A_{2}-\gamma_{2}\right)\left(\beta_{1}\left(A_{2}+\gamma_{2}\right)-\left(\alpha_{1}+\alpha_{2}\right)\right) .
\end{aligned}
$$

Now, (3.48) becomes

$$
\lambda_{2}=\frac{2 \beta_{1}\left(A_{2}-\gamma_{2}\right)\left(\beta_{1}\left(A_{2}+\alpha_{2}\right)-\left(\alpha_{1}+\alpha_{2}\right)\right)}{\left(\alpha_{1}-\alpha_{2}\right)^{2}+2 A_{2} \beta_{1}\left(\alpha_{2}-\alpha_{1}\right)+\beta_{1}^{2}\left(A_{2}^{2}-\gamma_{2}^{2}\right)},
$$

establishing the proof of the theorem. 
Now, we consider the special case of System (1.1) when $A_{2}=\gamma_{2}$.

In this case system (1.1) becomes

$$
\begin{aligned}
& x_{n+1}=\frac{\alpha_{1}+\beta_{1} x_{n}}{y_{n}}, \\
& y_{n+1}=\frac{\alpha_{2}+A_{2} x_{n}}{A_{2}+y_{n}},
\end{aligned}
$$

Equilibrium points are solutions of the following system:

$$
\begin{aligned}
& \bar{x}=\frac{\alpha_{1}+\beta_{1} \bar{x}}{\bar{y}}, \\
& \bar{y}=\frac{\alpha_{2}+A_{2} \bar{y}}{A_{2}+\bar{x}} .
\end{aligned}
$$

The second equation implies

$$
\bar{x}=\frac{\alpha_{2}-\alpha_{1}}{\beta_{1}}, \quad \alpha_{2}>\alpha_{1}
$$

Now, the first equation implies

$$
\bar{y}=\frac{\beta_{1} \alpha_{2}}{\alpha_{2}-\alpha_{1}}, \quad \alpha_{2}>\alpha_{1}
$$

The map $T$ associated to System (3.55) is given by

$$
T(x, y)=\left(\frac{\alpha_{1}+\beta_{1} x}{y}, \frac{\alpha_{2}+A_{2} y}{A_{2}+x}\right) .
$$

The Jacobian matrix of the map $T$ has the following form:

$$
\mathrm{J}_{T}=\left(\begin{array}{cc}
\frac{\beta_{1}}{y} & -\frac{\alpha_{1}+\beta_{1} x}{y^{2}} \\
-\frac{\alpha_{2}+A_{2} y}{\left(A_{2}+x\right)^{2}} & \frac{\gamma_{2}}{A_{2}+x}
\end{array}\right) .
$$

The value of the Jacobian matrix of $T$ at the equilibrium point is

$$
\mathrm{J}_{T}(\bar{x}, \bar{y})=\left(\begin{array}{cc}
\frac{\beta_{1}}{\bar{y}} & -\frac{\bar{x}}{\bar{y}} \\
-\frac{\bar{y}}{A_{2}+\bar{x}} & \frac{A_{2}}{A_{2}+\bar{x}}
\end{array}\right) .
$$


The determinant of (3.61) is given by

$$
\operatorname{det} \mathrm{J}_{T}(\bar{x}, \bar{y})=\frac{\beta_{1} A_{2}-\overline{x y}}{\bar{y}\left(A_{2}+\bar{x}\right)}
$$

The trace of (3.61) is

$$
\operatorname{Tr} \mathrm{J}_{T}(\bar{x}, \bar{y})=\frac{\beta_{1}}{\bar{y}}+\frac{A_{2}}{A_{2}+\bar{x}}
$$

Theorem 3.4. Assume

$$
A_{2}=\gamma_{2}, \quad \alpha_{2}>\alpha_{1}
$$

Then there exists a unique positive equilibrium point

$$
E=\left(\frac{\alpha_{2}-\alpha_{1}}{\beta_{1}}, \frac{\beta_{1} \alpha_{2}}{\alpha_{2}-\alpha_{1}}\right)
$$

of system (1.1), which is a saddle point. The following statements hold.

(a) If $\beta_{1} A_{2}-\alpha_{2}>0$, then $\lambda_{1} \in(1,+\infty)$ and $\lambda_{2} \in(0,1)$.

(b) If $\beta_{1} A_{2}-\alpha_{2}<0$, then $\lambda_{1} \in(1,+\infty)$ and $\lambda_{2} \in(-1,0)$.

Proof. We prove that $E$ is a saddle point.

We check the conditions

$$
\left|\operatorname{Tr} \mathrm{J}_{T}(\bar{x}, \bar{y})\right|>1+\operatorname{det} \mathrm{J}_{T}(\bar{x}, \bar{y}), \quad \operatorname{Tr}^{2} \mathrm{~J}_{T}(\bar{x}, \bar{y})-4 \operatorname{det} \mathrm{J}_{T}(\bar{x}, \bar{y})>0 .
$$

Condition $\left|\operatorname{Tr} \mathrm{J}_{T}(\bar{x}, \bar{y})\right|>1+\operatorname{det} \mathrm{J}_{T}(\bar{x}, \bar{y})$ is equivalent to

$$
\frac{\beta_{1}}{\bar{y}}+\frac{A_{2}}{A_{2}+\bar{x}}>1+\frac{\beta_{1} A_{2}}{\left(A_{2}+\bar{x}\right) \bar{y}}-\frac{\bar{x}}{A_{2}+\bar{x}}
$$

This implies

$$
\beta_{1}\left(A_{2}+\bar{x}\right)+A_{2} \bar{y}>\bar{y} A_{2}+\overline{x y}+\beta_{1} A_{2}-\overline{x y} \Longleftrightarrow \beta_{1} \bar{x}>0 .
$$

Condition

$$
\operatorname{Tr}^{2} \mathrm{~J}_{T}(\bar{x}, \bar{y})-4 \operatorname{det} \mathrm{J}_{T}(\bar{x}, \bar{y})>0
$$


is equivalent to

$$
\left(\frac{\beta_{1}}{\bar{y}}-\frac{\gamma_{2}}{A_{2}+\bar{x}}\right)^{2}+4 \frac{\overline{x y}}{\bar{y}\left(A_{2}+\bar{x}\right)}>0
$$

Hence $E$ is a saddle point.

Now,

$$
\begin{gathered}
\lambda_{1}+\lambda_{2}=\frac{\beta_{1}}{\bar{y}}, \\
\lambda_{1} \lambda_{2}=\frac{\beta_{1} A_{2}-\alpha_{2}}{\bar{y}\left(A_{2}+\bar{x}\right)} .
\end{gathered}
$$

The first equation implies that either both eigenvalues are positive or the smaller one is less then zero. The second equation implies that

$$
\begin{aligned}
& \text { If } \beta_{1} A_{2}>\alpha_{2} \text {, then } \lambda_{1} \in(1,+\infty), \lambda_{2} \in(0,-1) \text {; } \\
& \text { If } \beta_{1} A_{2}<\alpha_{2} \text {, then } \lambda_{1} \in(1,+\infty), \lambda_{2} \in(-1,0) \text {, }
\end{aligned}
$$

establishing the proof of theorem.

\section{Global Behavior}

Theorem 4.1. Assume

$$
A_{2}<\gamma_{2}
$$

Then system (1.1) has a unique equilibrium point $E_{1}$ which is a saddle point. Furthermore, there exists the global stable manifold $W^{s}\left(E_{1}\right)$ that separates the positive quadrant so that all orbits below this manifold are asymptotic to $(\infty, 0)$, and all orbits above this manifold are asymptotic to $(0, \infty)$. All orbits that start on $W^{s}\left(E_{1}\right)$ are attracted to $E_{1}$. The global unstable manifold $W^{u}\left(E_{1}\right)$ is the graph of a continuous, unbounded, strictly decreasing function.

Proof. The existence of the global stable manifold $W^{s}\left(E_{1}\right)$ with the stated properties follows from Theorems 1.5, 1.7, and 1.8 and Lemmas 2.1 and 2.2.

Theorem 4.2. Assume

$$
A_{2}>\gamma_{2}, \quad \beta_{1}\left(A_{2}-\gamma_{2}\right)<\alpha_{2}-\alpha_{1}, \quad\left[\left(\alpha_{1}-\alpha_{2}\right)+\beta_{1}\left(A_{2}-\gamma_{2}\right)\right]^{2}-4 \alpha_{1} \beta_{1}\left(A_{2}-\gamma_{2}\right)>0 .
$$

Then system (1.1) has two equilibrium points: $E_{1}$ which is a saddle point and $E_{2}$ which is a sink. Furthermore, there exists the global stable manifold $W^{s}\left(E_{1}\right)$ that separates the positive quadrant so that all orbits below this manifold are asymptotic to $(\infty, 0)$, and all orbits above this manifold are attracted to equilibrium $E_{2}$. All orbits that start on $W^{s}\left(E_{1}\right)$ are attracted to $E_{1}$. The global unstable 
manifold $W^{u}\left(E_{1}\right)$ is the graph of a continuous, unbounded, strictly decreasing function with end point $E_{2}$.

Proof. The existence of the global stable manifold $W^{s}\left(E_{2}\right)$ with the stated properties follows from Theorems 1.5, 1.7, and 1.8 and Lemmas 2.1 and 2.2.

Theorem 4.3. Assume

$$
A_{2}>\gamma_{2}, \quad \beta_{1}\left(A_{2}-\gamma_{2}\right)<\alpha_{2}-\alpha_{1}, \quad\left[\left(\alpha_{1}-\alpha_{2}\right)+\beta_{1}\left(A_{2}-\gamma_{2}\right)\right]^{2}-4 \alpha_{1} \beta_{1}\left(A_{2}-\gamma_{2}\right)=0 .
$$

Then system (1.1) has a unique equilibrium $E_{2}=E_{3}$ which is non-hyperbolic. The sequences $\left\{x_{2 n}\right\},\left\{x_{2 n+1}\right\},\left\{y_{2 n}\right\}$, and $\left\{y_{2 n+1}\right\}$ are eventually monotonic. Every solution that starts in $Q_{4}\left(E_{2}\right)$ is asymptotic to $(\infty, 0)$, and every solution that starts in $Q_{2}\left(E_{2}\right)$ is asymptotic to the equilibrium $E_{2}$. Furthermore, there exists the global stable manifold $W^{s}\left(E_{2}\right)$ that separates the positive quadrant into three invariant regions, so that all orbits below this manifold are asymptotic to $(\infty, 0)$, and all orbits that start above this manifold are attracted to the equilibrium $E_{2}$. All orbits that start on $W^{s}\left(E_{2}\right)$ are attracted to $E_{2}$.

Proof. The existence of the global stable manifold $W^{s}\left(E_{2}\right)$ with the stated properties follows from Theorems 1.5, 1.7, and 1.8 and Lemmas 2.1 and 2.2.

First we prove that for all points $M(x)=\left(x,\left(\alpha_{1} / x\right)+\beta_{1}\right), x \neq 0$, the following holds:

$$
M(x) \varliminf_{\mathrm{se}} T(M(x)) .
$$

Observe that $M(x)$ is actually an arbitrary point on the curve $x=\left(\alpha_{1}+\beta_{1} x\right) / y$, which represents one of two equilibrium curves for system (1.1).

Indeed,

$$
\begin{aligned}
T\left(x,\left(\alpha_{1} / x\right)+\beta_{1}\right) & =\left(x, \frac{\alpha_{2}+\gamma_{2}\left(\left(\alpha_{1} / x\right)+\beta_{1}\right)}{A_{2}+x}\right) \\
& =\left(x, \frac{\alpha_{2} x+\gamma_{2}\left(\alpha_{1}+\beta_{1} x\right)}{\left(A_{2}+x\right) x}\right) .
\end{aligned}
$$

Now we have

$$
\begin{aligned}
\left(x, \frac{\alpha_{1}}{x}+\beta_{1}\right) & \leq_{\text {se }}\left(x, \frac{\alpha_{2} x+\gamma_{2}\left(\alpha_{1}+\beta_{1} x\right)}{x\left(A_{2}+x\right)}\right) \\
& \Longleftrightarrow x \leq x, \quad \frac{\alpha_{1}+\beta_{1} x}{x} \geq \frac{\alpha_{2} x+\gamma_{2}\left(\alpha_{1}+\beta_{1} x\right)}{x\left(A_{2}+x\right)} .
\end{aligned}
$$

The last inequality is equivalent to

$$
\left(\alpha_{1}+\beta_{1} x\right)\left(A_{2}+x\right) \geq \alpha_{2} x+\gamma_{2} \alpha_{1}+\beta_{1} \gamma_{2} x
$$


This is equivalent to

$$
\beta_{1} x^{2}+x\left(\alpha_{1}-\alpha_{2}+\beta_{1}\left(A_{2}-\gamma_{2}\right)\right)+\alpha_{1}\left(A_{2}-\gamma_{2}\right) \geq 0,
$$

which always holds since the discriminant of the quadratic polynomial on the left-hand side is zero.

Note that $M(x) \varliminf_{\mathrm{se}} E_{2}$, and $M(x)=E_{2}$ for $x=\left(\left(\alpha_{2}-\alpha_{1}\right)-\beta_{1}\left(A_{2}-\gamma_{2}\right)\right) / 2 \beta_{1}$.

Monotonicity of the map $T$ implies

$$
T^{n}(M(x)) \preceq_{\mathrm{se}} T^{n+1}(M(x))
$$

Set $T^{n}(M(x))=\left\{\left(x_{n}, y_{n}\right)\right\}$. Then the sequence $\left\{x_{n}\right\}$ is increasing and bounded by $x$-coordinate of the equilibrium, and the sequence $\left\{y_{n}\right\}$ is decreasing and bounded by $y$-coordinate of the equilibrium. This implies that $\left\{\left(x_{n}, y_{n}\right)\right\}$ converges to the equilibrium as $n \rightarrow \infty$.

Now, take any point $A(x, y) \in Q_{2}\left(E_{2}\right)$. Then there exists point $M\left(x^{*}\right)$ such that $M\left(x^{*}\right) \leq_{\mathrm{se}} A(x, y) \leq_{\mathrm{se}} E_{2}$. By using monotonicity of the map $T$, we obtain

$$
T^{n}\left(M\left(x^{*}\right)\right) \varliminf_{\mathrm{se}} T^{n}(A(x, y)) \preceq_{\mathrm{se}} E_{2} .
$$

Letting $n \rightarrow \infty$ in (4.10), we have

$$
\lim _{n \rightarrow \infty} T^{n}(A(x, y))=E_{2}
$$

Now, we consider $Q_{4}\left(E_{2}\right)$. By choosing $M(x)$ such that $E_{2} \preceq_{\mathrm{se}} M(x)$, we note that

$$
E_{2} \preceq_{\mathrm{se}} M(x) \preceq_{\mathrm{se}} T(M(x))
$$

By using monotonicity of the map $T$, we have

$$
T^{n}(M(x)) \preceq_{\mathrm{se}} T^{n+1}(M(x))
$$

Set $T^{n}(M(x))=\left\{\left(x_{n}, y_{n}\right)\right\}$. Then the sequence $\left\{x_{n}\right\}$ is increasing, and the sequence $\left\{y_{n}\right\}$ is decreasing and bounded by $y$-coordinate of equilibrium and has to converge. If $\left\{x_{n}\right\}$ converges, then $\left\{\left(x_{n}, y_{n}\right)\right\}$ has to converge to the equilibrium, which is impossible. This implies that $x_{n} \rightarrow \infty, n \rightarrow \infty$. Since $y_{n+1}=\left(\alpha_{2}+\gamma_{2} y_{n}\right) /\left(A_{2}+x_{n}\right)$, then $\lim _{n \rightarrow \infty} y_{n}=0$.

Now, take any point $B(x, y)$ in $Q_{4}\left(E_{2}\right)$. Then there is point $M\left(x^{* *}\right)$ such that $E_{2} \preceq_{\mathrm{se}} M\left(x^{* *}\right) \preceq_{\mathrm{se}} B(x, y)$. Using monotonicity of the map $T$, we have

$$
E_{2} \preceq_{\mathrm{se}} T^{n}\left(M\left(x^{* *}\right)\right) \preceq_{\mathrm{se}} T^{n}(B(x, y))
$$

Since, $T^{n}\left(M\left(x^{* *}\right)\right)$ is asymptotic to $(\infty, 0)$, then $\lim _{n \rightarrow \infty} T^{n}(B(x, y))=(\infty, 0)$. 
Theorem 4.4. Assume

$$
A_{2}=\gamma_{2}, \quad \alpha_{2}>\alpha_{1} .
$$

Then system (1.1) has a unique equilibrium $E$ which is a saddle point. Furthermore, there exists the global stable manifold $W^{s}(E)$ that separates the positive quadrant so that all orbits below this manifold are asymptotic to $(\infty, 0)$, and all orbits above this manifold are asymptotic to $(0, \infty)$. All orbits that start on $W^{s}(E)$ are attracted to $E$. The global stable manifold $W^{u}(E)$ is the graph of a continuous, unbounded, strictly increasing function.

Proof. The existence of the global stable manifold $W^{s}(E)$ with the stated properties follows from Theorems 1.5, 1.7, and 1.8 and Lemmas 2.1 and 2.2.

Theorem 4.5. Assume

$$
\begin{gathered}
A_{2}>\gamma_{2}, \quad\left[\left(\alpha_{1}-\alpha_{2}\right)+\beta_{1}\left(A_{2}-\gamma_{2}\right)\right]^{2}-4 \alpha_{1} \beta_{1}\left(A_{2}-\gamma_{2}\right)<0, \\
A_{2}>\gamma_{2}, \quad\left[\left(\alpha_{1}-\alpha_{2}\right)+\beta_{1}\left(A_{2}-\gamma_{2}\right)\right]^{2}-4 \alpha_{1} \beta_{1}\left(A_{2}-\gamma_{2}\right) \geq 0, \quad \beta_{1}\left(A_{2}-\gamma_{2}\right)>\alpha_{2}-\alpha_{1}
\end{gathered}
$$

or

$$
A_{2}=\gamma_{2}, \quad \alpha_{2} \leq \alpha_{1} .
$$

Then system (1.1) does not possess an equilibrium point. Its global behavior is described as follows:

$$
x_{n} \longrightarrow \infty, \quad y_{n} \longrightarrow 0, \quad n \longrightarrow \infty
$$

Proof. If the conditions of this theorem are satisfied, then (2.6) implies that there is no real (if the first condition of this theorem is satisfied) or positive equilibrium points (if the second condition of this theorem is satisfied).

Consider the second equation of system (1.1). That is,

$$
y_{n+1}=\frac{\alpha_{2}+\gamma_{2} y_{n}}{A_{2}+x_{n}}
$$

Note the following

$$
y_{n+1} \leq \frac{\alpha_{2}}{A_{2}}+\frac{\gamma_{2}}{A_{2}} y_{n}
$$

Now, consider equation

$$
u_{n+1}-\frac{\gamma_{2}}{A_{2}} u_{n}=\frac{\alpha_{2}}{A_{2}} .
$$


Its solution is given by

$$
u_{n}=c\left(\frac{\gamma_{2}}{A_{2}}\right)^{n}+\frac{\alpha_{2}}{A_{2}-\gamma_{2}}
$$

Since $A_{2}>\gamma_{2}$, then letting $n \rightarrow \infty$ we obtain that $u_{n} \rightarrow 0$. Now, (4.21) implies

$$
y_{n} \leq \frac{\alpha_{2}}{A_{2}-\gamma_{2}}+\varepsilon, \quad n \longrightarrow \infty
$$

This means that sequence $\left\{y_{n}\right\}$ is bounded for $A_{2}>\gamma_{2}$.

In order to prove the global behavior in this case, we decompose System (1.1) into the system of even-indexed and odd-indexed terms as

$$
\begin{aligned}
x_{2 n+1} & =\frac{\alpha_{1}+\beta_{1} x_{2 n}}{y_{2 n}}, \\
x_{2 n} & =\frac{\alpha_{1}+\beta_{1} x_{2 n-1}}{y_{2 n-1}}, \\
y_{2 n+1} & =\frac{\alpha_{2}+\gamma_{2} y_{2 n}}{A_{2}+x_{2 n}}, \\
y_{2 n} & =\frac{\alpha_{2}+\gamma_{2} y_{2 n-1}}{A_{2}+x_{2 n-1}},
\end{aligned}
$$

for $n=1,2, \ldots$

Lemma 2.1 implies that subsequences $\left\{x_{2 n+1}\right\},\left\{x_{2 n}\right\},\left\{y_{2 n+1}\right\}$, and $\left\{y_{2 n}\right\}$ are eventually monotone.

Since sequence $\left\{y_{n}\right\}$ is bounded, then the subsequences $\left\{y_{2 n+1}\right\}$ and $\left\{y_{2 n}\right\}$ must converge. If the sequences $\left\{x_{2 n+1}\right\}$ and $\left\{x_{2 n}\right\}$ would converge to finite numbers, then the solution of (1.1) would converge to the period-two solution, which is impossible by Lemma 2.2. Thus at least one of the subsequences $\left\{x_{2 n+1}\right\}$ and $\left\{x_{2 n}\right\}$ tends to $+\infty, n \rightarrow \infty$. Assume that $x_{2 n} \rightarrow \infty$ as $n \rightarrow \infty$. In view of third equation of (4.25), $y_{2 n+1} \rightarrow 0$, and in view of first equation of (4.25), $x_{2 n+1} \rightarrow \infty$ which by fourth equation of (4.25) implies that $y_{2 n+1} \rightarrow 0$ as $n \rightarrow \infty$.

Now, we prove the case when $A_{2}=\gamma_{2}$ and $\alpha_{2}=\alpha_{1}$.

In this case System (1.1) becomes

$$
\begin{aligned}
& x_{n+1}=\frac{\alpha_{1}+\beta_{1} x_{n}}{y_{n}}, \\
& y_{n+1}=\frac{\alpha_{1}+A_{2} y_{n}}{A_{2}+x_{n}} .
\end{aligned}
$$

The map $T$ associated to System (4.26) is given by

$$
T(x, y)=\left(\frac{\alpha_{1}+\beta_{1} x}{y}, \frac{\alpha_{1}+A_{2} y}{A_{2}+x}\right) .
$$


Equilibrium curves $C_{1}$ and $C_{2}$ can be given explicitly as the following functions of $x$ :

$$
\begin{aligned}
& C_{1}: y_{1}=\frac{\alpha_{1}}{x}+\beta_{1}, \\
& C_{2}: y_{2}=\frac{\alpha_{1}}{x} .
\end{aligned}
$$

It is obvious that these two curves do not intersect, which means that System (4.26) does not possess an equilibrium point.

Similarly, as in the proof of Theorem 4.3 , for all points $C_{1}(x)=\left(x,\left(\alpha_{1} / x\right)+\beta_{1}\right), x \neq 0$ the following holds:

$$
C_{1}(x) \preceq T\left(C_{1}(x)\right)
$$

Indeed,

$$
\begin{aligned}
T\left(x, \frac{\alpha_{1}}{x}+\beta_{1}\right) & =\left(x, \frac{\alpha_{1}+A_{2}\left(\frac{\alpha_{1}}{x}+\beta_{1}\right)}{A_{2}+x}\right) \\
& =\left(x, \frac{\alpha_{1} x+A_{2}\left(\alpha_{1}+\beta_{1} x\right)}{\left(A_{2}+x\right) x}\right) .
\end{aligned}
$$

Now, we have

$$
\begin{aligned}
\left(x, \frac{\alpha_{1}}{x}+\beta_{1}\right) & \unlhd_{\mathrm{se}}\left(x, \frac{\alpha_{1} x+A_{2}\left(\alpha_{1}+\beta_{1} x\right)}{\left(A_{2}+x\right) x}\right) \\
& \Longleftrightarrow x \leq x \text { and } \frac{\alpha_{1}}{x}+\beta_{1} \geq \frac{\alpha_{1} x+A_{2}\left(\alpha_{1}+\beta_{1} x\right)}{\left(A_{2}+x\right) x} .
\end{aligned}
$$

The last inequality is equivalent to

$$
\left(\alpha_{1}+\beta_{1} x\right)\left(A_{2}+x\right) \geq \alpha_{1} x+A_{2} \alpha_{1}+\beta_{1} A_{2} x
$$

which always holds.

Monotonicity of $T$ implies

$$
T^{n}\left(C_{1}(x)\right) \varliminf_{\mathrm{se}} T^{n+1}\left(C_{1}(x)\right)
$$

Set $T^{n}\left(C_{1}(x)\right)=\left\{\left(x_{n}^{\prime}, y_{n}^{\prime}\right)\right\}$. Then the sequence $\left\{x_{n}^{\prime}\right\}$ is increasing and the sequence $\left\{y_{n}^{\prime}\right\}$ is decreasing. Since $\left\{y_{n}^{\prime}\right\}$ is decreasing and $y_{n}^{\prime}>0, n=1,2, \ldots$, then it has to converge. If $\left\{x_{n}^{\prime}\right\}$ converges, then $\left\{\left(x^{\prime}{ }_{n}, y_{n}^{\prime}{ }_{n}\right)\right\}$ has to converge to the equilibrium, which is impossible. This implies that $x_{n}^{\prime} \rightarrow \infty, n \rightarrow \infty$. The second equation of System (4.26) implies that $y_{n}^{\prime} \rightarrow 0, n \rightarrow \infty$. 
Now, take any point $(x, y) \in \mathbb{R}_{+}^{2}$. Then there exists point $C_{1}\left(x^{\star}\right)=\left(x^{\star}, \alpha_{1} / x^{\star}\right)$ such that

$$
C_{1}\left(x^{\star}\right) \varliminf_{\mathrm{se}}(x, y)
$$

Monotonicity of $T$ implies

$$
T^{n}\left(C_{1}\left(x^{\star}\right) \varliminf_{\mathrm{se}} T^{n}(x, y) .\right.
$$

Set

$$
T^{n}(x, y)=\left(x_{n}, y_{n}\right), \quad T^{n}\left(C_{1}\left(x^{\star}\right)\right)=\left(x_{n}^{\prime \prime}, y_{n}^{\prime \prime}\right)
$$

Then, we have

$$
\begin{aligned}
& x_{n}^{\prime \prime} \leq x_{n} \\
& y_{n}^{\prime \prime} \geq y_{n} .
\end{aligned}
$$

Since

$$
x_{n}^{\prime \prime} \longrightarrow \infty, \quad y_{n}^{\prime \prime} \longrightarrow 0, \quad n \longrightarrow \infty
$$

we conclude, using the inequalities (4.37), that

$$
x_{n} \longrightarrow \infty, \quad y_{n} \longrightarrow 0, \quad n \longrightarrow \infty
$$

Similarly, we can prove the case $A_{2}=\gamma_{2}, \alpha_{2}<\alpha_{1}$.

\section{References}

[1] E. Camouzis, M. R. S. Kulenović, G. Ladas, and O. Merino, "Rational systems in the plane," Journal of Difference Equations and Applications, vol. 15, no. 3, pp. 303-323, 2009.

[2] M. R. S. Kulenović and O. Merino, "Global bifurcation for discrete competitive systems in the plane," Discrete and Continuous Dynamical Systems. Series B, vol. 12, no. 1, pp. 133-149, 2009.

[3] M. R. S. Kulenović and O. Merino, "Invariant manifolds for competitive discrete systems in the plane," to appear in International Journal of Bifurcation and Chaos, http:/ /arxiv.org/abs/0905.1772v1.

[4] Dž. Burgić, S. Kalabušić, and M. R. S. Kulenović, "Nonhyperbolic dynamics for competitive systems in the plane and global period-doubling bifurcations," Advances in Dynamical Systems and Applications, vol. 3, no. 2, pp. 229-249, 2008.

[5] Dž. Burgić, M. R. S. Kulenović, and M. Nurkanović, "Global dynamics of a rational system of difference equations in the plane," Communications on Applied Nonlinear Analysis, vol. 15, no. 1, pp. 71-84, 2008.

[6] D. Clark and M. R. S. Kulenović, "A coupled system of rational difference equations," Computers $\mathcal{E}$ Mathematics with Applications, vol. 43, no. 6-7, pp. 849-867, 2002.

[7] D. Clark, M. R. S. Kulenović, and J. F. Selgrade, "Global asymptotic behavior of a two-dimensional difference equation modelling competition," Nonlinear Analysis. Theory, Methods \& Applications, vol. 52, no. 7, pp. 1765-1776, 2003. 
[8] J. E. Franke and A.-A. Yakubu, "Mutual exclusion versus coexistence for discrete competitive systems," Journal of Mathematical Biology, vol. 30, no. 2, pp. 161-168, 1991.

[9] J. E. Franke and A.-A. Yakubu, "Geometry of exclusion principles in discrete systems," Journal of Mathematical Analysis and Applications, vol. 168, no. 2, pp. 385-400, 1992.

[10] M. Garić-Demirović, M. R. S. Kulenović, and M. Nurkanović, "Global behavior of four competitive rational systems of difference equations in the plane," Discrete Dynamics in Nature and Society, vol. 2009, Article ID 153058, 34 pages, 2009.

[11] M. W. Hirsch and H. Smith, "Monotone dynamical systems," in Handbook of Differential Equations: Ordinary Differential Equations. Vol. II, pp. 239-357, Elsevier, Amsterdam, The Netherlands, 2005.

[12] M. R. S. Kulenović and O. Merino, "Competitive-exclusion versus competitive-coexistence for systems in the plane," Discrete and Continuous Dynamical Systems. Series B, vol. 6, no. 5, pp. 1141-1156, 2006.

[13] M. R. S. Kulenović and M. Nurkanović, "Asymptotic behavior of a system of linear fractional difference equations," Journal of Inequalities and Applications, no. 2, pp. 127-143, 2005.

[14] M. R. S. Kulenović and M. Nurkanović, "Asymptotic behavior of a competitive system of linear fractional difference equations," Advances in Difference Equations, vol. 2006, Article ID 19756, 13 pages, 2006.

[15] H. L. Smith, "Invariant curves for mappings," SIAM Journal on Mathematical Analysis, vol. 17, no. 5, pp. 1053-1067, 1986.

[16] H. L. Smith, "Periodic competitive differential equations and the discrete dynamics of competitive maps," Journal of Differential Equations, vol. 64, no. 2, pp. 165-194, 1986.

[17] H. L. Smith, "Periodic solutions of periodic competitive and cooperative systems," SIAM Journal on Mathematical Analysis, vol. 17, no. 6, pp. 1289-1318, 1986.

[18] H. L. Smith, "Planar competitive and cooperative difference equations," Journal of Difference Equations and Applications, vol. 3, no. 5-6, pp. 335-357, 1998.

[19] H. L. Smith, "Non-monotone systems decomposable into monotone systems with negative feedback," Journal of Differential Equations, vol. 53, pp. 747-758, 2006.

[20] P. de Mottoni and A. Schiaffino, "Competition systems with periodic coefficients: a geometric approach," Journal of Mathematical Biology, vol. 11, no. 3, pp. 319-335, 1981. 